SUBJECT AREAS:

NON-SMALL-CELL LUNG

CANCER

TARGETED THERAPIES

INSULIN SIGNALLING

PRE-CLINICAL STUDIES

Received

14 March 2013

Accepted

31 July 2013

Published

2 September 2013

Correspondence and requests for materials should be addressed to

J.A.M. (jmenendez@

iconcologia.net;

jmenendez@idibgi.

org)

* These authors contributed equally to this work.

\section{transition (EMT) crosstalk suppresses the erlotinib-sensitizing effect of EGFR exon 19 deletion mutations}

Alejandro Vazquez-Martin ${ }^{1,2 *}$, Sílvia Cufi ${ }^{1}, 2 *$, Cristina Oliveras-Ferraros ${ }^{1,2 *}$, Violeta Zenobia Torres-Garcia ${ }^{1,2}$, Bruna Corominas-Faja ${ }^{1,2}$, Elisabet Cuyàs' ${ }^{1,2}$, Rosa Bonavia ${ }^{3}$, Joana Visa ${ }^{3}$, Begoña Martin-Castillo ${ }^{2,4}$, Enrique Barrajón-Catalán ${ }^{5,6}$, Vicente Micol ${ }^{5,6}$, Joaquim Bosch-Barrera ${ }^{2,7}$ \& Javier A. Menendez ${ }^{1,2}$

${ }^{1}$ Metabolism \& Cancer Group, Translational Research Laboratory, Catalan Institute of Oncology, Girona, Catalonia (SPAIN), ${ }^{2}$ Girona Biomedical Research Institute (IDIBGi), Girona, Catalonia, Spain, ${ }^{3}$ Animal Care Facility, IDIBELL, L'Hospitalet de Llobregat, Barcelona, Catalonia, Spain, ${ }^{4}$ Unit of Clinical Research, Catalan Institute of Oncology, Girona, Catalonia, Spain, ${ }^{5}$ Molecular and Cellular Biology Institute (IBMC), Miguel Hernández University, Elche, Alicante, Spain, ${ }^{6}$ Monteloeder, Inc., Elche, Alicante, Spain,

${ }^{7}$ Medical Oncology, Catalan Institute of Oncology, Girona, Catalonia, Spain.

Using non-small cell lung carcinoma (NSCLC) cells harboring the erlotinib-sensitizing Epidermal Growth Factor Receptor (EGFR) exon 19 mutation delE746-A750, we developed erlotinib-refractory derivatives in which hyperactive Insulin-like Growth Factor-1 Receptor (IGF-1R) signaling associated with enrichment in epithelial-to-mesenchymal transition (EMT)-related morphological and transcriptional features. We then explored whether an IGF-1R/EMT crosstalk was sufficient to promote erlotinib refractoriness in the absence of second-site EGFR mutations, MET and AXL hyperactivation. Transforming Growth Factor-beta1 (TGFß1)-induced mesenchymal trans-differentiation was sufficient to impede erlotinib functioning in the presence of drug-sensitive delE746-A750 EGFR mutation. Pharmacological blockade of IGF-1R fully prevented the TGFß1's ability to activate an EMT protein signature [E-cadherin low/vimentin high]. The sole presence of erlotinib was capable of rapidly activate an IGF-1R-dependent, vimentin-enriched mesenchymal-like phenotype in delE746-A750-mutated epithelial cells. Even if transient, NSCLC cells' intrinsic plasticity to undergo crosstalk between IGF-1R and EMT signaling pathways can sufficiently eliminate the erlotinib-sensitizing effect of highly prevalent EGFR mutations and suggests the urgent need for dual IGF-1R/EMT-targeting strategies to circumvent erlotinib resistance.

omatic mutations in exons encoding the tyrosine kinase (TK) domain of the epidermal growth factor receptor (EGFR) are positively associated with increased sensitivity of non-small cell lung cancer (NSCLC) patients to the selective EGFR TK inhibitors (TKIs) gefitinib (Iressa ${ }^{\mathrm{TM}}$ ) and erlotinib $\left(\text { Tarceva }^{\mathrm{TM}}\right)^{1-4}$. Prospective trials have demonstrated an impressive $\sim 75 \%$ response rate for patients whose tumors harbor these mutations ${ }^{5,6}$, which occur as either multinucleotide in-frame deletions in exon 19 (e.g., delE746-A750) or as single missense mutations (e.g., L858R). Unfortunately, the EGFR TKI-driven improvement in patient outcome is notably limited because virtually all NSCLC patients with erlotinib/gefitinib-sensitizing EGFR mutations eventually acquire resistance after a median of 6-12 months of EGFR TKI therapy ${ }^{7,8}$. In approximately half of cases, the tumor cells present after disease progression contain second-site mutations that alter drug binding to the EGFR TK domain" ${ }^{9-13}$. The most common lesion $(>90 \%)$ is the so-called "gatekeeper mutation," which involves a substitution of methionine for threonine at position 790 (T790M).

More recently, in addition to other second-site mutations associated with acquired resistance to EGFR TKIs (e.g., L747S and D761Y in exon 19 or T854A in exon 21), novel EGFR-independent mechanisms have been discovered that contribute to EGFR TKI resistance either in the absence or presence of the EGFR T790M mutation reviewed in 14-16. The second well-known mechanism of gefitinib/erlotinib resistance is the MET receptor tyrosine kinase (RTK) gene amplification. MET creates a bypass signaling track that activates AKT through 
HER3-mediated activation of PI3K in the presence of EGFR TKIs ${ }^{17}$. Although approximately $60 \%$ of $M E T$ amplifications are independent of the T790M mutation, MET amplification and EGFR T790M are not mutually exclusive; they can be detected in the same resistant tumor or may occur independently in different metastatic sites in the same patient ${ }^{17-19}$; it remains to be unambiguously demonstrated whether treatment with EGFR TKIs selects for pre-existing cells with the EGFR T790M mutation and/or MET amplification during the acquisition of EGFR TKI resistance or whether resistant tumors are emerged during the treatment.

EGFR T790M and MET amplification, which can be detected in up to $20 \%$ of EGFR NSCLCs secondarily refractory to EGFR TKIs, account for approximately $60-70 \%$ of all known causes of acquired resistance to gefitinib/erlotinib. Consequently, ongoing research is attempting to identify the mechanisms that may account for the 30$40 \%$ of EGFR TKI-resistant, EGFR-mutated tumors that do not carry $E G F R$ mutations or MET amplification. Although increased activation of insulin-like growth factor-1 receptor (IGF-1R) through the loss of IGF-binding proteins and loss or reduction of the tumor suppressor PTEN have been associated with acquired resistance to EGFR TKIs in laboratory models ${ }^{20-22}$, these mechanisms have not yet been validated in specimens from EGFR TKI-refractory patients. Activation of the AXL RTK through overexpression or upregulation of the AXL ligand GAS6 confers acquired resistance to erlotinib in pre-clinical models of EGFR-mutated NSCLCs; AXL is overexpressed in $\sim 20 \%$ of post-erlotinib-treated patients with EGFR TKI resistance ${ }^{23}$. Mutations in PIK3CA have been identified in $\sim 5 \%$ of EGFR-mutant lung cancers with acquired resistance to EGFR TKIs ${ }^{24}$. Interestingly, AXL upregulation has been associated with the development of an epithelial-to-mesenchymal transition (EMT) ${ }^{23}$. Similarly, genetic and histological analyses of tumor biopsies from NSCLCs with acquired resistance to EGFR inhibitors revealed that a subgroup of resistant carcinomas underwent a pronounced EMT ${ }^{24}$. EMT, which has been largely considered a general biological feature rendering NSCLCs sensitive or insensitive to EGFR inhibition ${ }^{25-28}$, has also been associated with acquired resistance to EGFR TKIs in laboratory models ${ }^{29,30}$. Development of EMT was observed in a NSCLC patient who acquired resistance to erlotinib in the absence of known resistance mechanisms, such as the EGFR T790M mutation and MET amplification ${ }^{30}$. In this scenario, it is tempting to suggest that EMT is a convergent mechanism that might be rapidly selected to bypass the EGFR pathways in resistant tumors.

Here, using EGFR-mutated PC-9 NSCLC cells, we developed erlotinib-refractory PC-9 derivatives lacking most of the mechanisms of secondary resistance described to date. We now present proof-ofconcept evidence that in the absence of second-site EGFR mutations, MET hyperactivation, PI3KCA mutation or activation of AXL, the stimulation of crosstalk between IGF-1R and EMT signaling pathways, even if transient, is sufficient to notably suppress the erlotinibsensitizing effect of the highly-prevalent EGFR exon 19 in-frame deletion mutation in lung carcinoma cells.

\section{Results}

PC-9 cells with acquired resistance to erlotinib cross-activate the insulin-like growth factor-1 receptor IGF-1R. Human EGFR Pathway qBiomarker Somatic Mutation PCR Arrays, which combine allele-specific amplification and $5^{\prime}$ hydrolysis probe detection to detect as little as $0.01 \%$ somatic mutation in a background of wildtype genomic DNA, were used to assess the status of 85 possible mutations in the EGFR pathway. The array likewise detected the erlotinib-sensitizing deletion mutation delE746-A750 in EGFR exon 19 in parental PC-9 cells. Of note, we failed to detect new cooccurring mutations in the EGFR pathway when the pooled populations of PC-9 cells with acquired resistance to erlotinib, which maintained the same expression level of EGFR delE746$A 750$, were profiled in parallel (data not shown).
After excluding the presence of a secondary resistance mutation in the EGFR gene, we investigated whether aberrant activation of another RTKs such as MET or AXL, might mediate erlotinib resistance. We took advantage of a low-scale phospho-proteomic approach to molecularly profile RTKs that might distinctively correlate with PC-9 sensitivity/resistance to erlotinib. To simultaneously assess the activation status of multiple receptor tyrosine kinases (RTKs), we employed a commercially available phospho-proteome assay [Human Phospho-RTK Array Proteome Profiler ${ }^{\mathrm{TM}}$ ], which allows for the rapid, sensitive, and simultaneous detection of 42 RTKs in a single antibody-based microarray. This protein array technology allows for screening of multiple phospho-proteins without performing numerous immunoprecipitation and/or immunoblotting analyses. Erlotinib-responsive PC-9 parental cells and their erlotinib-refractory PC-9/ErlR derivatives were treated with erlotinib and then immediately lysed and analyzed for changes in phosphorylated signaling responses. Because the lysates were collected immediately following erlotinib exposure, the results likely represent immediate responses by tumor cells to erlotinib. In untreated PC-9 cells, EGFR (HER1) was the sole highly phosphorylated receptor among the four members of the HER network, and this EGFR phosphorylation was notably decreased in the presence of erlotinib (Fig. 1A). Phospho-RTK profiling of cell lysates from untreated PC-9/ErlR POOLs clearly revealed that the constitutive status of EGFR phosphorylation was mostly unaltered compared with parental PC-9 cells. PC-9/ErlR POOLs notably displayed an erlotinib-refractory activation of EGFR accompanied by hyperactivation of the insulin growth factor-1 receptor (IGF-1R) (Fig. 1A).

MET, AXL, and HER2 were among the RTKs tested in the phospho-RTK array; therefore, our results confirmed that acquired resistance in non-small cell lung cancer cells bearing the erlotinibsensitizing deletion mutation delE746-A750 in EGFR exon 19 may occur in the absence of most of the known mechanisms of secondary resistance, including second-site EGFR mutations, MET and/or AXL activation, HER2 amplification or loss of the mutant delE746-A750 EGFR gene itself. By using Human EGFR Pathway qBiomarker Somatic Mutation PCR Arrays we confirmed also that acquired resistance to erlotinib took place in the absence of secondary $B R A F$ and/ or NRAS mutations (data not shown).

Pharmacological inhibition of IGF-1R activity circumvents acquired resistance to erlotinib. The above results strongly suggested that EGFR and IGF-1R might cooperate to endow delE746-A750-mutated PC-9 cells with higher pro-survival signaling in response to erlotinib. To test this hypothesis, we compared the effects of erlotinib alone or in combination with I-OMe-AG538, a cell-permeable analog of tyrphostin AG538 and potent inhibitor of the IGF-1R kinase activity. Accordingly, when used in combination with erlotinib, I-OMeAG538 was sufficient to sensitize cells to the growth-inhibitory activity of erlotinib (Fig. 1B). The ability of I-OMe-AG538 to overcome or circumvent acquired resistance to erlotinib was more striking in long-term clonogenic assays than short-term MTT assays (data not shown), indicating that the combinatorial treatment not only altered the kinetics of cell survival/cell death but also decreased the number of cells that survived the combination treatment to subsequently proliferate and form colonies once the co-treatment ceased. Thus, whereas erlotinib and I-OMe-AG538 as single agents slightly altered the anchorage-dependent colony-forming ability of erlotinib-refractory PC-9/ErlR pooled populations, a 3-day concurrent treatment with erlotinib and $10 \mu \mathrm{mol} / \mathrm{L}$ I-OMe-AG538 was sufficient to markedly suppress the frequency of surviving cells when colony formation was assessed 10 days later (Fig. 1B). Equivalent results were found when the more specific inhibitor of IGF-1R AG1024 substituted I-OMe-AG538. Thus, a 10-fold lower concentration of AG1024 -i.e., $1 \mu \mathrm{mol} / \mathrm{L}$ - was sufficient to 

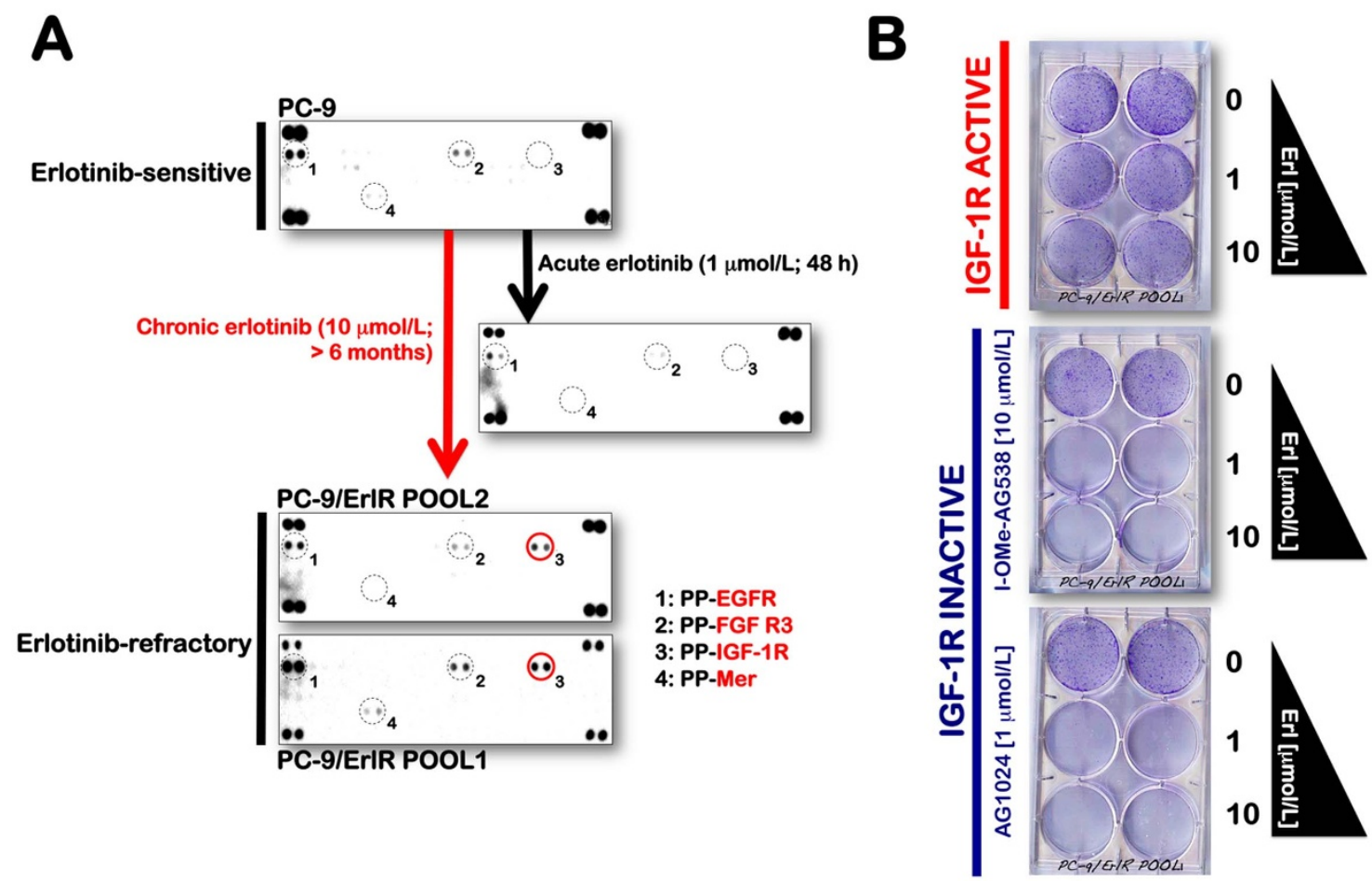

Figure $1 \mid$ Activation of IGF-1R contributes to erlotinib resistance. (A). Phosphoproteome profiling of PC-9 cells before and after acquisition of resistance to erlotinib. Total cell lysates $(750 \mu \mathrm{g})$ from PC-9 parental cells before and after adaptation (POOLs) to erlotinib treatment were incubated with membranes of the phosphoproteomic platform human Phospho-RTK including 42 different RTKs, as per the manufacturer's instructions (Proteome Profiler; R\&D Systems). Figure shows representative phosphoproteome analyses that were developed on X-ray film following exposure to chemiluminescent reagents. Equivalent results were obtained in three independent experiments. Of the 42 RTKs analyzed via phospho-arrays, IGF-1R underwent the highest induction in phosphorylation when comparing erlotinib-refractory PC-9 ErlR pools to erlotinib-sensitive PC-9 parental cells. (B). Clonogenic survival assay of erlotinib-refractory PC-9/ErlR cells treated with graded concentrations of erlotinib in the absence or presence of the IGF1R inhibitors I-Ome-AG538 or AG1024, as specified. Representative photographs show the crystal violet-stained colonies of PC-9/ErlR POOL1 cells given different treatments either alone or in combination (in duplicate) in a 6-well plate. (Erl: Erlotinib).

synergistically circumvent erlotinib-resistance in PC-9/ErlR pooled populations (Fig. 1B).

The sub-cellular localization of phospho-IGF-1R suggests the involvement of the epithelial-to-mesenchymal transition (EMT) phenomenon during the acquisition of resistance to erlotinib. We evaluated whether enhanced signaling from IGF-1R in erlotinibrefractory PC-9 pooled populations occurred in distinct subcellular compartments linked to EMT-like features. We performed high-content immunofluorescence microscopy studies to visualize sub-cellular accumulation of the active form of IGF-1R using an antibody against phospho-IGF-1R at Tyr1161 (Fig. 2). Of note, we observed a striking cell membrane-associated local accumulation of the active form of IGF-1R in erlotinib-refractory PC-9 pooled populations but not in erlotinib-responsive PC-9 parental cells (Fig. 2, top). Examination of phospho-IGF-1R ${ }^{\text {Tyrl161 }}$ under a higher magnification revealed that the active form of IGF-1R was distinctly distributed at the tips of the numerous cell protrusions found in erlotinib-refractory PC-9 pools (Fig. 2, bottom). Because the subcellular distribution of phospho-IGF-1R $\mathrm{R}^{\text {Tyr1161 }}$ indicated a possible association of IGF-1R with the EMT-like process, we performed co-staining experiments with antibodies directed against phosphoIGF-1R $\mathrm{R}^{\mathrm{Tyr} 161}$ and the cytoskeletal protein F-actin (Fig. 2). Erlotinibrefractory PC-9 cells exhibited a drastic reorganization of the cytoskeleton compared with erlotinib-sensitive PC-9 parental cells. Whereas erlotinib-responsive PC-9 cells contained a homogeneous distribution of F-actin label, a dramatic reorganization of F-actin into stress fibers that emanated from phospho-IGF-1R $\mathrm{R}^{\mathrm{Tyr} 1161}$-positive focal adhesion-like structures were clearly recognizable in erlotinib-refractory PC-9/ErlR pooled populations. Thus, most of erlotinib-refractory PC-9 cells displayed dendritic-like protrusions with a concentration of F-actin at the phospho-IGF-1 $\mathrm{R}^{\mathrm{Tyr} 161}$-positive tips. Indeed, co-staining experiments with the cytoskeletal protein vinculin supported the concentration of phospho-IGF-1R $\mathrm{R}^{\mathrm{Tyr} 1161}$ at the edges of profuse and numerous focal adhesion-like structures in the cell membrane of PC-9 cells with acquired resistance to erlotinib (Fig. 3, left).

PC-9 cells with acquired resistance to erlotinib activate an EMT transcriptional program. The above-mentioned morphological alterations in erlotinib-refractory PC- 9 pooled populations were compatible with an execution of a genetic program of cells undergoing EMT, which is defined by the rapid transcriptional repression of epithelial markers and sustained induction of EMTpromoting transcription factors. To assess whether EMT-related morphological changes in the erlotinib-refractory PC-9 pooled populations were transcriptionally regulated by events directly controlling the EMT phenomenon, the total RNA from erlotinibsensitive PC-9 parental cells and erlotinib-refractory PC-9 cells was evaluated using a customized array for the quantitative real-time PCR (qRT-PCR) analysis of 19 genes specifically associated with EMT and the reciprocal mesenchymal-to-epithelial transition (see the "Materials and Methods" section for the genes included in the array). When a two-fold or greater difference in the mRNA expression level was used as the cut-off to determine significant regulatory events for the genes involved in the activation and maintenance of EMT phenomena, erlotinib-refractory PC-9 cells were found to have significantly increased mRNA levels of a 

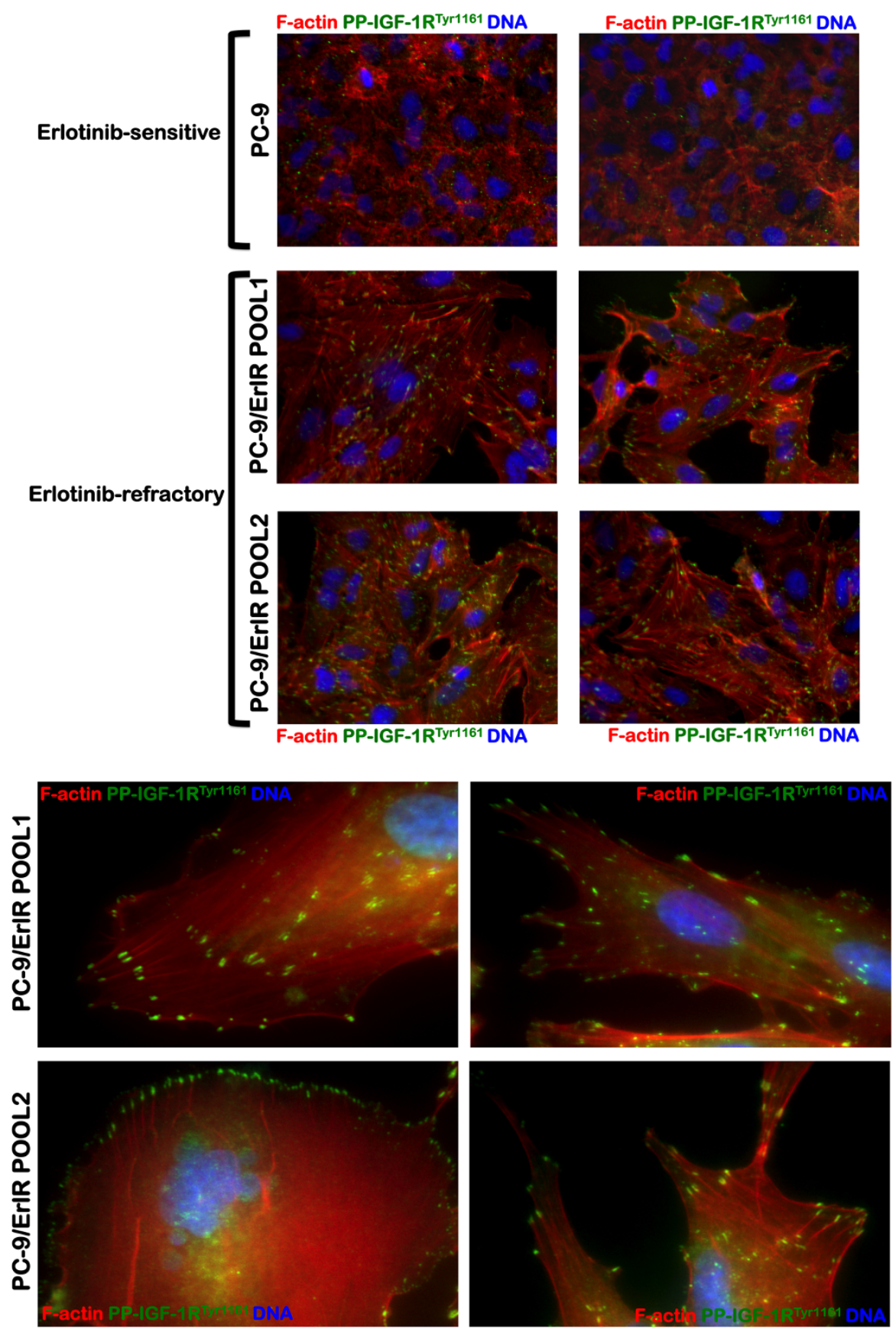

Figure $2 \mid$ Cytoskeleton organization and distribution of the active form of IGF-1R in erlotinib-refractory PC-9 cells. After fixation and permeabilization, cellular co-distribution of PP-IGF-1 $\mathrm{R}^{\mathrm{Tyr} 1161}$ and F-actin was assessed following staining with anti-PP-IGF-1R ${ }^{\mathrm{Tyr} 1161}$ and anti-F-actin antibodies, as specified, and Hoechst 33258 for nuclear counterstaining. Images show representative portions of erlotinib-sensitive PC-9 parental cells and erlotinib-refractory PC-9 POOLs captured in different channels for PP-IGF-1R ${ }^{\text {Tyr1161 }}$ (green), F-actin (red), and Hoechst 33258 (blue) with a $20 \times$ objective, and merged on BD Pathway 855 Bioimager System using BD Attovision software.

significant subset of EMT-inducing transcription factors (TFs) (Fig. 3, right). The EMT TFs significantly upregulated in erlotinibrefractory PC-9 cells included the homeobox protein goosecoid (GSC), the E-box-binding proteins $Z E B 1$ and $Z E B 2$, the zinc-finger proteins SNAIL1 and SLUG (SNAIL2), the basis helix-loop-helix protein TWIST1, and the transmembrane adhesion receptor for hyaluronan CD44.

In the absence of well-recognized mechanisms of acquired resistance to erlotinib, hyperactive IGF-1R signaling associated with a drastic enrichment in EMT-related morphological and transcriptional features appears to be sufficient to explain, at least in part, erlotinib unresponsiveness in the presence of the highly prevalent erlotinib-sensitizing delE746-A750 EGFR mutation.

Exogenous supplementation with TGFß1 induces an EMT-like phenomenon in delE746-A750-mutated PC-9 cells. The EMT gene expression profile was markedly altered in erlotinib-resistant PC-9 cells with activated IGF-1R compared with erlotinibresponsive $\mathrm{PC}-9$ parental cells, suggesting that the phenotypic appearance of erlotinib-refractory PC-9 cell populations is a result of progressive EMT changes. However, it was unclear whether the mesenchymal-like cells in the acquired-resistance cancer cell 


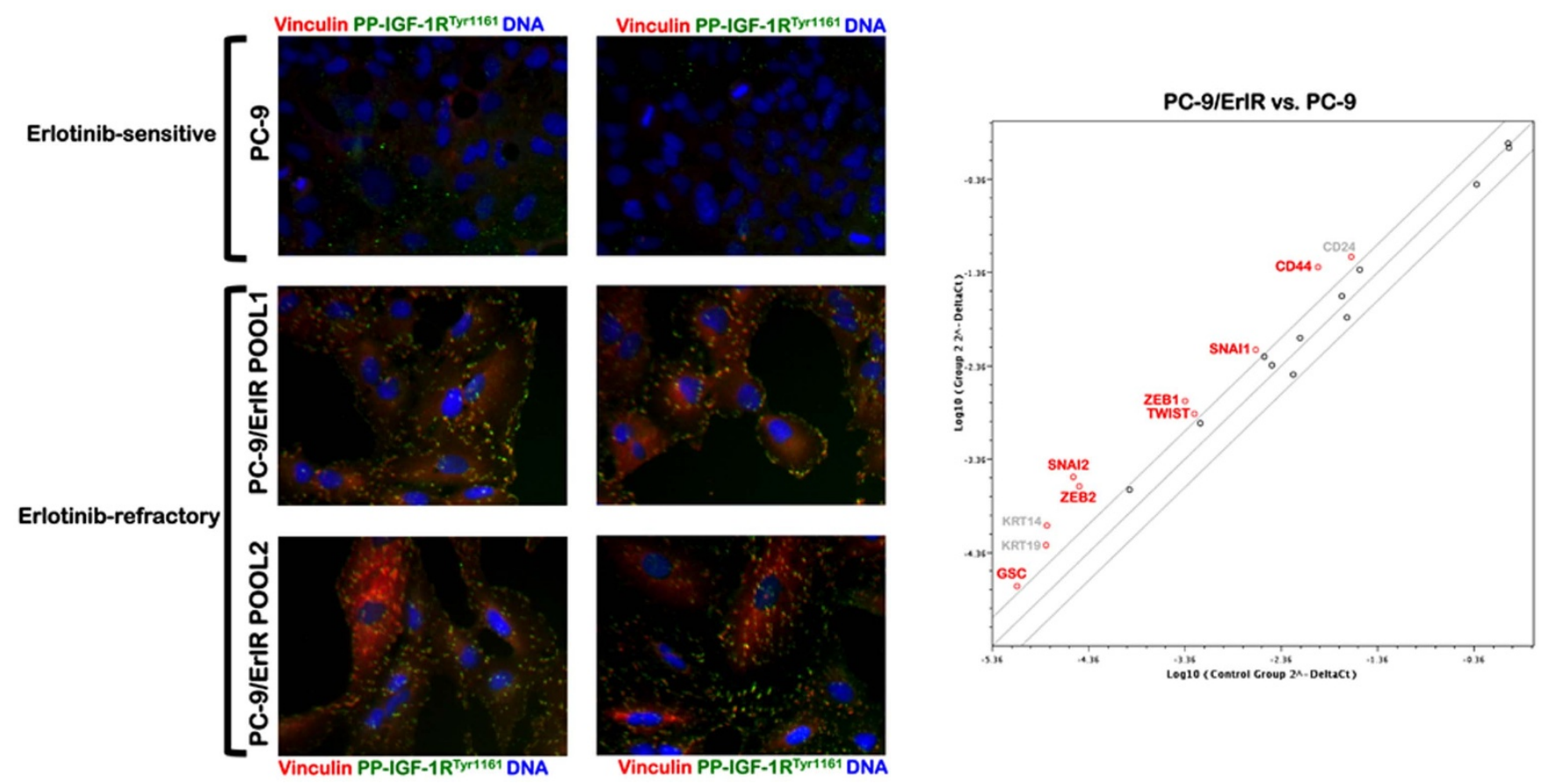

Figure $3 \mid$ A correlation between IGF-1R activation at focal adhesion-like structures and EMT transcriptomic signatures in erlotinib-refractory PC-9 cells. Left. After fixation and permeabilization, cellular co-distribution of PP-IGF-1R ${ }^{\text {Tyr1 } 161}$ and vinculin was assessed following staining with anti-PPIGF-1R $\mathrm{R}^{\mathrm{Tyr} 1161}$ and anti-vinculin antibodies, as specified, and Hoechst 33258 for nuclear counterstaining. Images show representative portions of erlotinibsensitive PC-9 parental cells and erlotinib-refractory PC-9 POOLs captured in different channels for PP-IGF-1R ${ }^{\text {Tyr1 161 }}$ ( green), vinculin (red), and Hoechst 33258 (blue) with a $20 \times$ objective, and merged on BD Pathway 855 Bioimager System using BD Attovision software. Right. EMT expression profiling of erlotinib-refractory PC-9 pooled cells versus erlotinib-sensitive PC-9 parental cells. Figure shows a representative scatter plot of the difference in the relative abundance of 19 mRNAs whose levels, in published results, are significantly altered during activation/deactivation of the EMT genetic program. Green and red symbols ( $\geq 2$-fold) denote downregulation and upregulation versus basal expression in PC-9 parental cells, respectively; grey symbols denote fold-change results that need to be validated with a sufficient number of biological replicates (i.e., fold-change results may have greater variation of $p$ value $>0.05$ or the $p$ value for the fold-change is either unavailable or relatively high $[p>0.05]$ or, alternatively, they are uninterpretable because the gene's average threshold cycles were either not determined or greater than the defined cut-off value [default 35] in both samples).

population existed prior to therapy or whether they were induced by erlotinib treatment. In this scenario, we sought to determine whether solely inducing EMT in PC-9 parental cells during or before exposure to erlotinib was sufficient to fully induce a cell phenotype unresponsive to erlotinib. We first aimed to confirm that treatment of PC-9 parental cells with the EMT inducer TGF $\beta 1$ was sufficient to promote cellular changes (i.e., morphology and size) resembling those of tumor cells undergoing a bona fide EMT genetic program. Untreated delE746-A750-mutated PC-9 cells exhibited a typical epithelial-like morphology. Upon treatment with TGF $\beta 1$ (10 ng/mL; $72 \mathrm{~h})$, PC-9 cells acquired a spindle-like morphology and notably reduced the number of cell-cell contacts (Fig. 4A, left). EMT-like alterations began after $24 \mathrm{~h}$, and they were maximal after $72 \mathrm{~h}$, when exposure to TGF $\beta 1$ fully induced a complete conversion of parental PC-9 epithelial cells to a fibroblast-like spindle-shaped elongated PC-9 mesenchymal morphology. In view of these findings suggesting that exposure to TGF $\beta 1$ efficiently triggered cellular changes consistent with EMT induction in delE746-A750-mutated PC-9 cells, we examined Factin reorganization during the mesenchymal-like conversion of PC-9 lung epithelial cancer cells following TGF $\beta 1$ administration. Exogenous TGF $\beta 1$ supplementation promoted a drastic redefinition of cell shape and morphology, including a dramatic formation of long stress fibers of F-actin filaments, which was accompanied by a noteworthy cytoplasmic accumulation of mesenchymal markers, such as the intermediate filament vimentin (Fig. 4A, right). These phenotypic changes were reversed by removal of TGF $\beta 1$ from the culture media (data not shown).
The transient induction of mesenchymal-like phenotypes with a minimal EMT-mRNA/protein signature [SLUG high/E-cadherin low] is sufficient to induce full refractoriness to erlotinib. We examined the effects of TGF $\beta 1$-induced transient EMT on cellular sensitivity to erlotinib (Fig. 5). PC-9/TGF $\beta 1$ cells were completely refractory to the growth inhibitory effects of erlotinib compared with parental PC-9 cells. Following TGF $\beta 1$-induced EMT, we failed to achieve half-maximal effective concentrations of erlotinib in PC-9 cells (Fig. 5). We next asked whether the changes in signaling axes used in mesenchymal-like PC9 cells with acquired resistance to erlotinib could be initiated by an EMT-like transition of epithelial PC9 parental cells simultaneously exposed to erlotinib. Concurrent exposure of erlotinib-treated cells to TGF $\beta 1$ notably reduced erlotinib-induced reduction in PC-9 cell viability (Fig. 5). To determine whether the EMT-like induced changes in the PC-9/ TGF $\beta 1$ model might somewhat (early) reflect those observed in (late) established mesenchymal-like PC-9/ErlR pooled populations, we measured the expression of the same set of EMT marker genes using qRT-PCR. When a two-fold or greater difference in the mRNA expression level was used again as the cut-off to determine significant EMT-related regulatory effects of short-term exposure to TGF $\beta 1$, the PC-9/TGF $\beta 1$ cells showed significantly increased levels of SLUG (SNAIL2) (Fig. 4B, left). Because SLUG is a member of the zincfinger transcription factor family that binds the E-cadherin $(C D H 1)$ gene promoter region to repress $\mathrm{CDH} 1$ transcription during EMT, it was not entirely unexpected to find that TGF $\beta 1$ induced up-regulation of SLUG was accompanied by significantly reduced transcription of the epithelia-specific gene markers $C D H 1$ 
PC-9

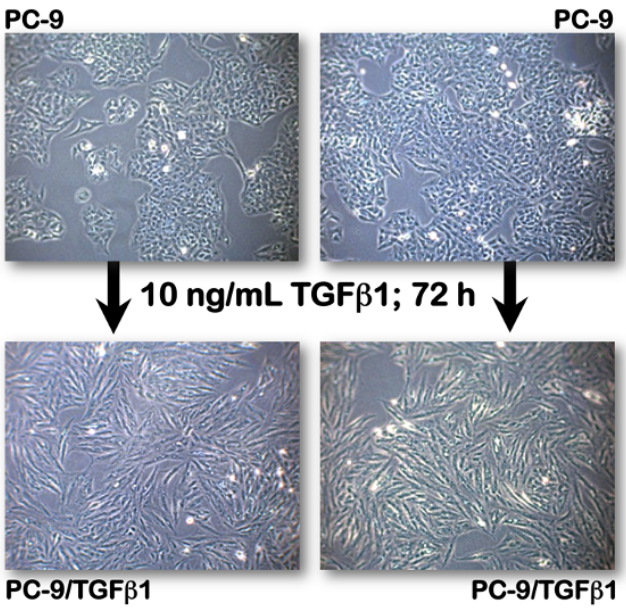

B

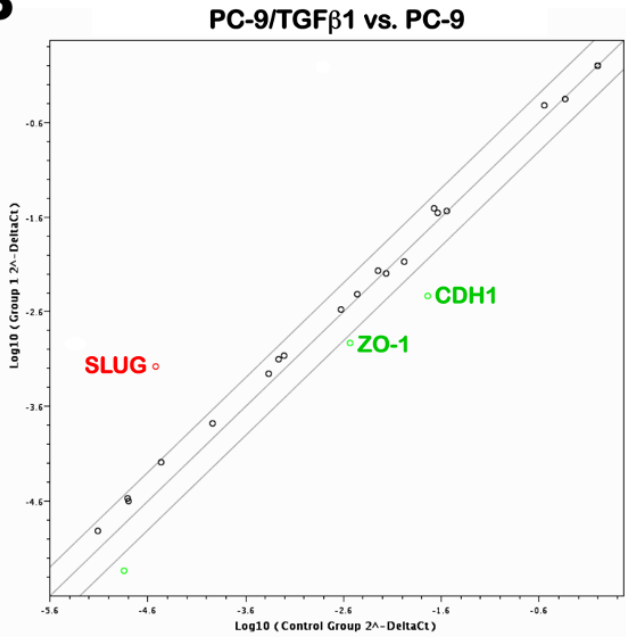

DNA F-actin Vimentin
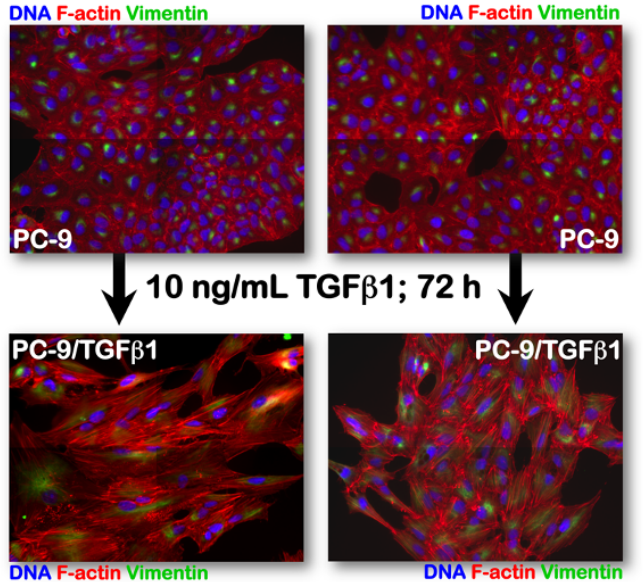

DNA E-Cadherin
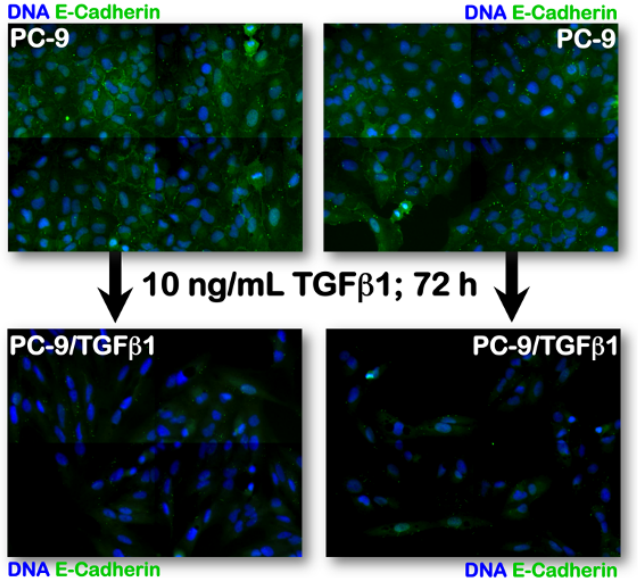

Figure 4 TGFß1 induces a bona fide EMT in erlotinib-sensitive PC-9 cells. (A). PC-9 cells were grown until 75\%-80\% confluence, serum-starved for $24 \mathrm{~h}$, and then treated for 3 days with $10 \mathrm{ng} / \mathrm{mL}$ TGF $\beta 1$. Phase-contrast (left) and immunofluorescence (right) images were obtained and merged on BD Pathway 855 Bioimager System according the recommended assay procedure (BD Attovision software). Images show representative portions $(\mathrm{n}=3)$ of untreated- or TGF $\beta 1$-treated PC-9 cells captured in different channels for F-actin (red), vimentin (green), and Hoechst 33258 (blue) with a $20 \times$ objective. (B). Left. EMT expression profiling of PC-9/TGF $\beta 1$ cells versus untreated PC-9 cells. Figure shows a representative scatter plot of the difference in the relative abundance of 19 mRNAs whose levels, in published results, are significantly altered during activation/deactivation of the EMT genetic program (see legend in Fig. 3). Right. After fixation and permeabilization, sub-cellular distribution of the epithelial marker E-cadherin was assessed following staining with an anti-E-cadherin antibody and Hoechst 33258 for nuclear counterstaining. Images show representative portions of untreated-and TGF $\beta 1$-treated PC-9 cells captured in different channels for E-cadherin (green) and Hoechst 33258 (blue) with a $20 \times$ objective, and merged on BD Pathway 855 Bioimager System using BD Attovision software.

and OCLN (occludin or zonula occludens-1 [ZO-1]). Immunostaining studies confirmed that the expected expression of E-cadherin in epithelial PC-9 cells was significantly altered in response to TGF $\beta 1$ treatment (Fig. 4B, right). While the epithelial cell marker Ecadherin was easily observable in untreated PC-9 control cells, TGF $\beta 1$-treated PC9 cells exhibited a drastically altered distribution of E-cadherin from a conspicuous, highly membranous staining to a more diffuse, barely detectable cytoplasmic staining.

Transient trans-differentiation to mesenchymal-like phenotypes through short-term exposure to TGF $\beta 1$, which activated a minimal EMT-mRNA/protein signature, including activation of the master EMT gene SLUG and suppression of the epithelial marker E-cadherin, was sufficient to induce unresponsiveness of delE746-A750mutated cells to erlotinib.

The induction of an erlotinib-desensitizing EMT phenomenon proceeds through the activation of IGF-1R. We aimed to explore whether the induction of EMT might proceed through the activation of IGF-1R to promote erlotinib refractoriness in the presence of EGFR exon 19 deletion mutations. Upon TGF $\beta 1$ treatment, PC-9 likewise flatted and spread very quickly and the morphological modifications to mesenchymal-like states were not associated with cell division. A remarkable reorganization of the active form of IGF$1 \mathrm{R}$ at the tips of the prominent F-actin stress fibers was clearly recognizable during TGF $\beta 1$-induced EMT in PC-9 cells (Fig 6); remarkably, the EMT-related re-localization of phospho-active IGF-1R to EMT-related sub-cellular structures was fully preventable when treatment with the EMT inducer TGF $\beta 1$ was performed in the presence of the IGF-1R inhibitor AG1024 (Fig. 6). AG1204 concurrently impeded also the TGF $\beta 1$ 's ability to drastically affect the constant cycling of E-cadherin between the cytoplasm and the cell membrane to promote that E-cadherin could be internalized faster than it is readdressed to the membrane; indeed, the visible loss of cell-cell contacts, the rapid internalization of 


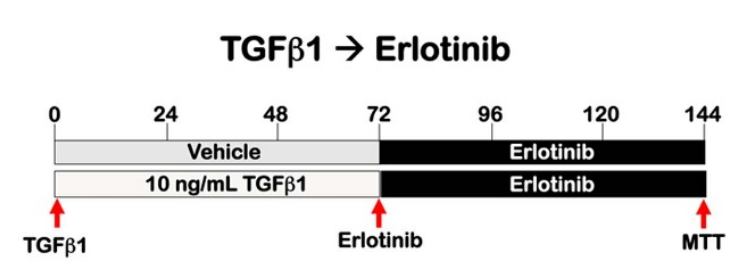

DNA F-actin Vimentin
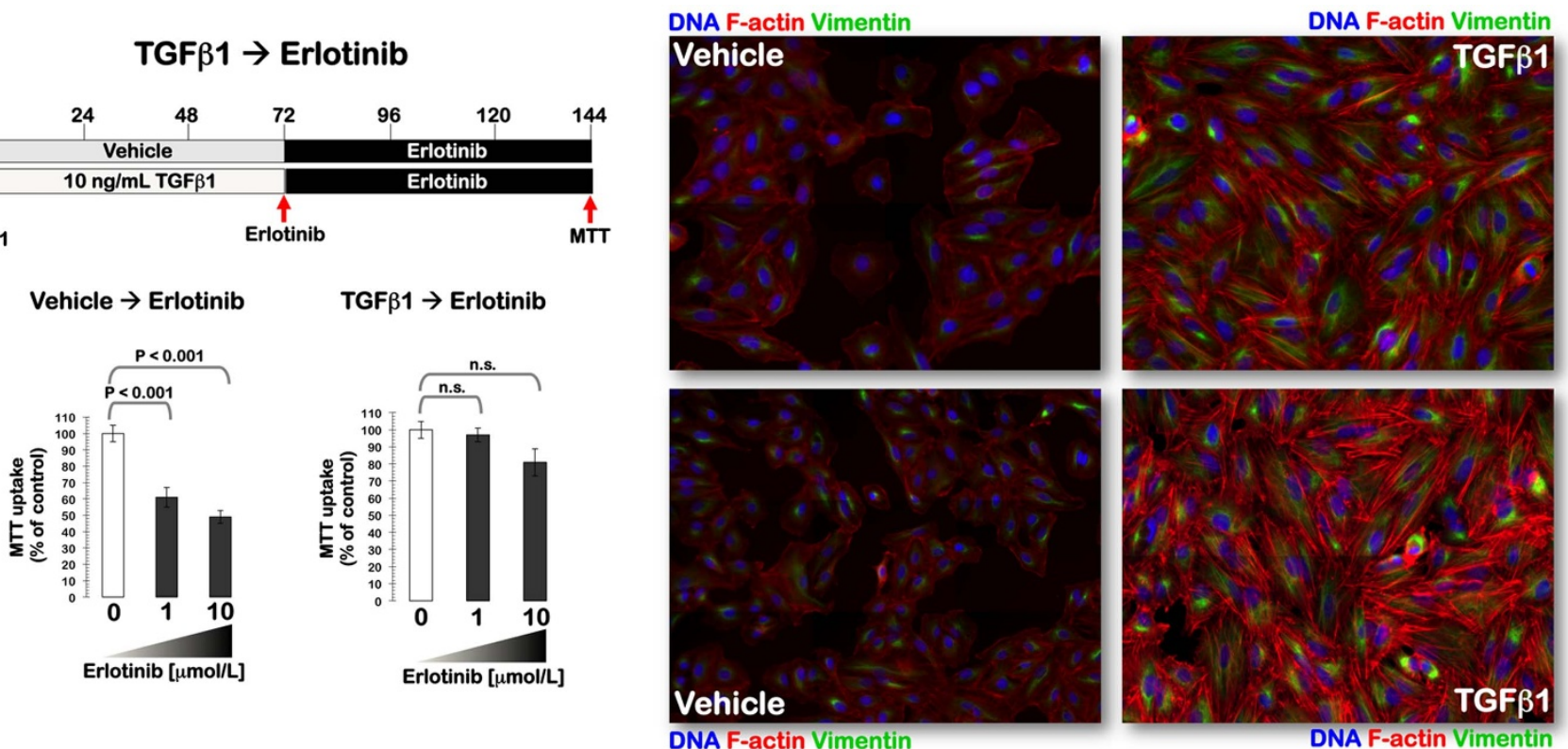

Erlotinib + TGF $\beta 1$
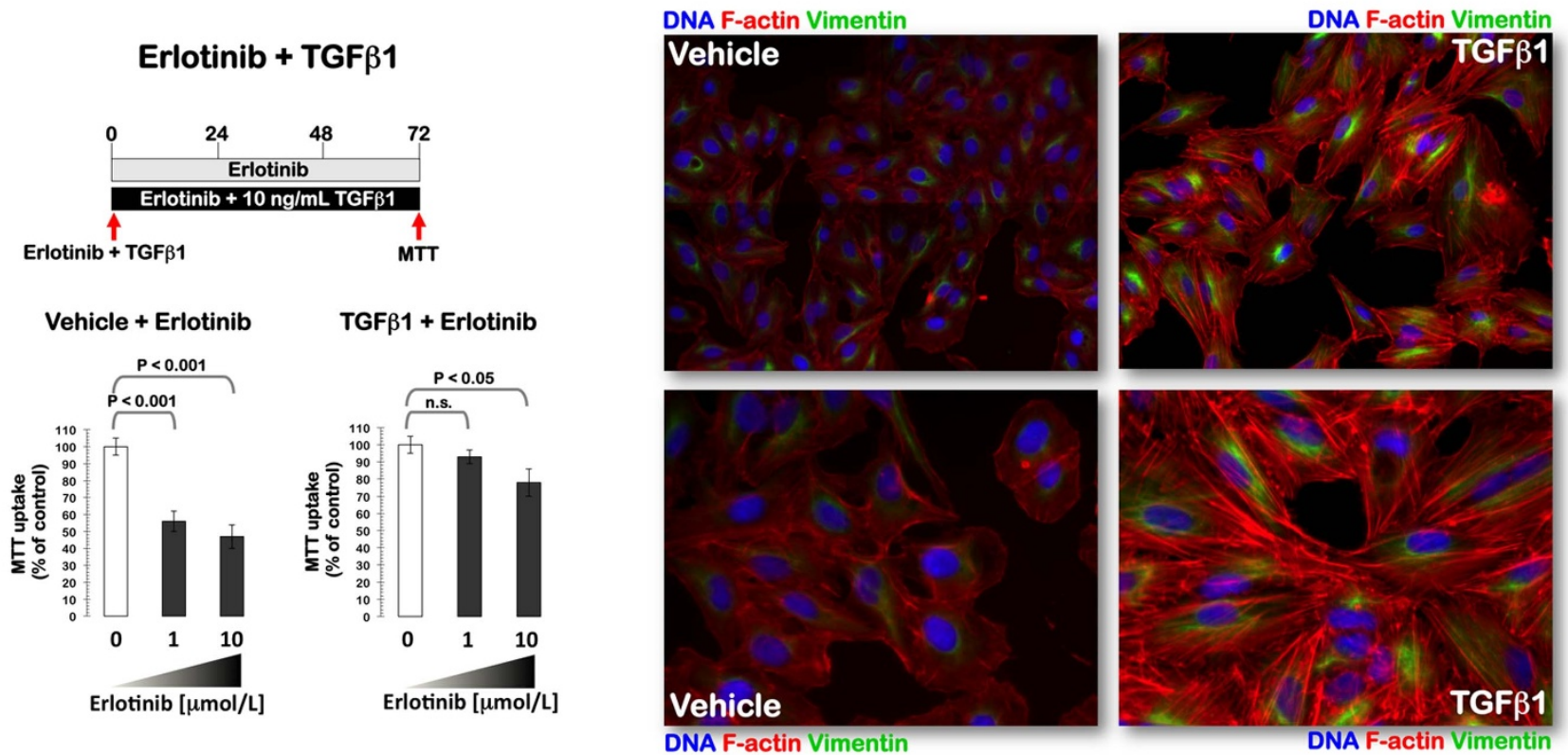

Figure 5 | TGFß1-induced EMT is sufficient to promote refractoriness to erlotinib in PC-9 cells. Left panels. The effects of sequential (top) or concurrent (bottom) treatment with the EMT inducer TGF $\beta 1$ on cell viability of PC-9 cells exposed to erlotinib were measured by MTT uptake assays. Figure shows dose-response graphs as $\%$ of untreated cell populations (untreated control cells $=100 \%$ cell viability). Results are means (columns) and $95 \%$ confidence intervals (bars) of three independent experiments made in triplicate. Statistically significant differences (one-factor ANOVA analysis) between MTT uptakes in treated and untreated control cells are shown. Statistical tests were two-sided. Right panels. Images show representative portions $(\mathrm{n}=3)$ of control- or TGF $\beta 1$-treated PC-9 cell cultures at the start of erlotinib treatment (top) or once the treatment with erlotinib is finished (bottom) and captured in different channels for F-actin (red), vimentin (green), and Hoechst 33258 (blue) with a $20 \times$ objective, and merged on BD Pathway 855 Bioimager System using BD Attovision software.

E-cadherin, and the neo-expression of the mesenchymal-specific marker vimentin were all prevented by AG1024 (Fig. 6). Therefore, pharmacological blockade of IGF-1R activity appeared to spatiotemporally control over the TGF $\beta 1$-driven pro-EMT signaling. During TGF $\beta 1$-induced EMT, we were able to achieve half-maximal effective concentrations of erlotinib when PC-9/ TGF $\beta 1$ cells were co-treated with the IGF-1R inhibitor AG1204 (Supplementary Fig. 1).

Short-term exposure to erlotinib is sufficient to enhance IGF-1Rdriven EMT-like features in delE746-A750-mutated PC-9 cells.
Although the above-mentioned findings might support the notion that TGF $\beta 1$ and IGF1R function in a linear fashion to drive loss of responsiveness to erlotinib in delE746-A750-mutated PC- 9 cells, the previously unrecognized ability of erlotinib to converge on the activation of an IGF-1R-driven EMT phenomenon might also be considered. We then assessed whether the sole presence of erlotinib was sufficient to activate an IGF-1R-activated/mesenchymallike phenotype in delE746-A750-mutated PC-9 epithelial cells. Intriguingly, short-term exposure to erlotinib significantly impacted on the epithelial morphology of PC-9 cells. After exposure to erlotinib, PC-9 cells became more loosely arranged and they 

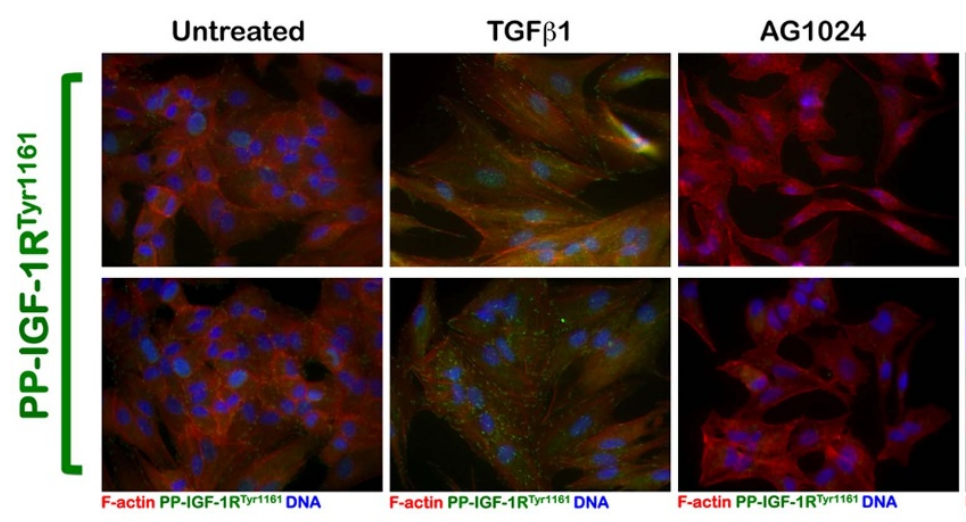

TGF $\beta 1+A G 1024$
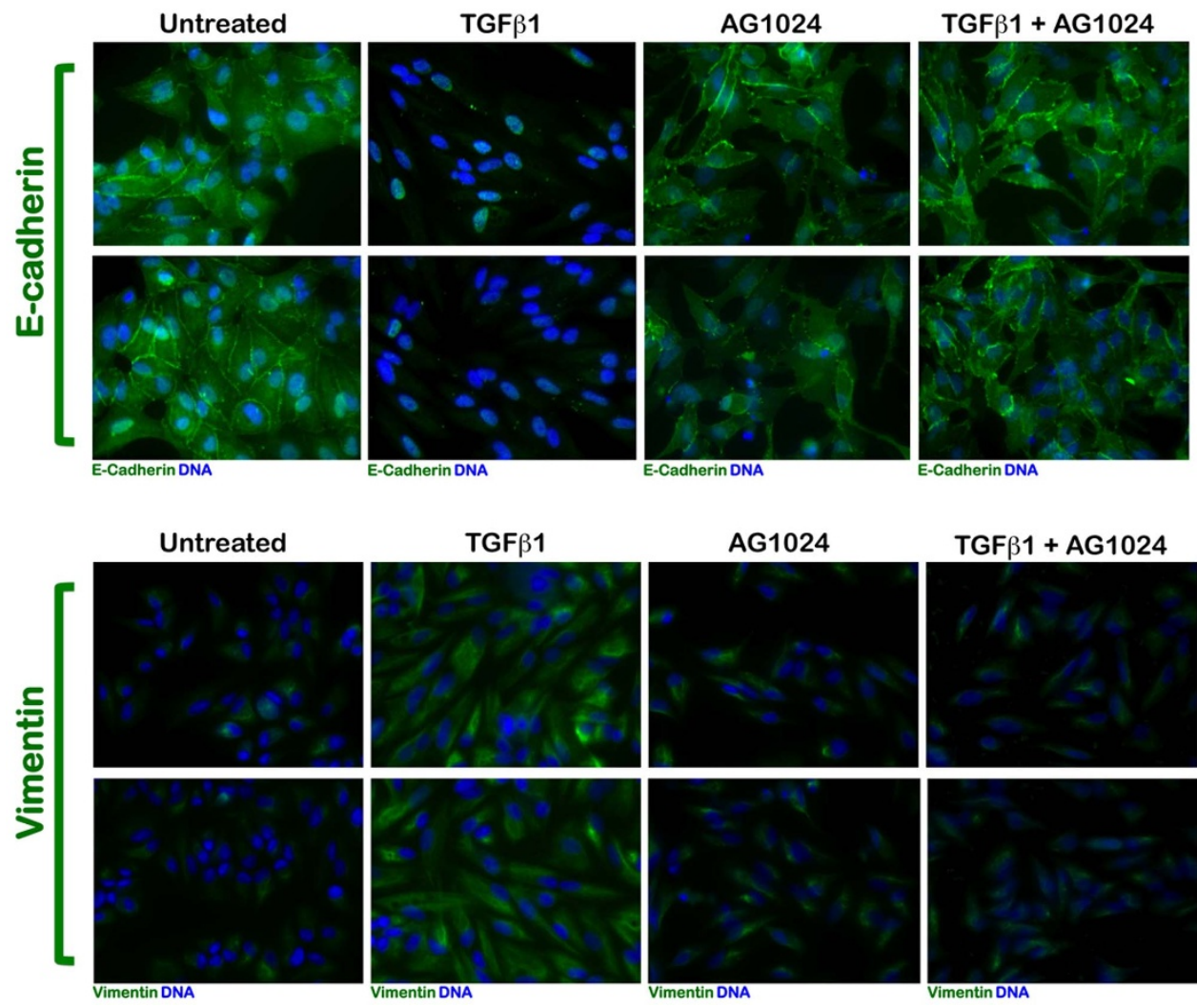

TGF $\beta 1+A G 1024$

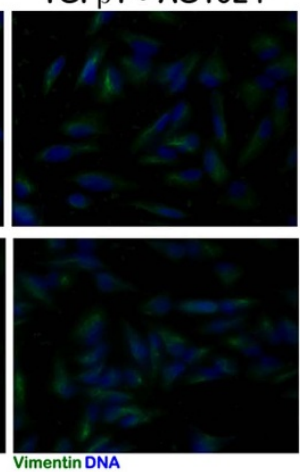

Figure 6 | Pharmacological inhibition of IGF-1R activity impedes TGF $\beta 1$-induced EMT in PC-9 cells. PC-9 cells were grown until 75\%-80\% confluence, serum-starved for $24 \mathrm{~h}$, and then treated for 3 days with $10 \mathrm{ng} / \mathrm{mL}$ TGF $\beta 1$ in the absence of presence of $1 \mu \mathrm{mol} / \mathrm{L}$ AG1024. Top panels. After fixation and permeabilization, cellular co-distribution of PP-IGF- $1 \mathrm{R}^{\mathrm{Tyr} 1161}$ and F-actin was assessed following staining with anti-PP-IGF-1R ${ }^{\mathrm{Tyr} 1161}$ and anti-F-actin antibodies, as specified, and Hoechst 33258 for nuclear counterstaining. Middle panels. After fixation and permeabilization, sub-cellular distribution of the epithelial marker E-cadherin was assessed following staining with an anti-E-cadherin antibody and Hoechst 33258 for nuclear counterstaining. Bottom panels. After fixation and permeabilization, sub-cellular distribution of the mesenchymal-specific marker vimentin was assessed following staining with an anti-vimentin antibody and Hoechst 33258 for nuclear counterstaining. Images show representative portions of untreated-,

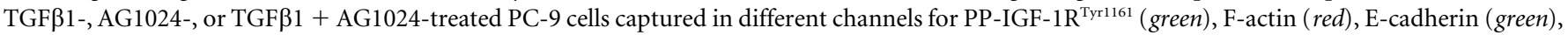
vimentin (green), and Hoechst 33258 (blue) with a $20 \times$ objective, and merged on BD Pathway 855 Bioimager System using BD Attovision software.

started to elongate. These changes progressed gradually to a spindleshaped morphology, which was evident at $\sim 24 \mathrm{~h}$ with erlotinib, although cells elongated further at $48 \mathrm{~h}$ and beyond. Changes in cell morphology corresponded with partial reorganization of filamentous actin (F-actin). In untreated PC-9 cells maintained in the absence of erlotinib, phalloidin-labeled F-actin was likewise predominantly organized in cortical bundles tightly associated with cell-cell adhesions. In contrast, after $72 \mathrm{~h}$ with erlotinib, Factin in subsets of cells was assembled into thick parallel bundles, or actin stress fibers, traversing the ventral cell surface (Fig. 7). Of note, the cells showing the most prominent erlotinib-induced enhancement of stress fibers concurrently exhibited a prominent re-localization of the phosphorylation of the IGF-1R at the tips of actin stress fibers. Short-term exposure to erlotinib appeared to activate a bona fide EMT transcriptional program because erlotinib-induced dynamic changes in cell morphology and actin filament organization were accompanied by a gradual decrease in the abundance of the epithelial cell-cell adhesion protein E-cadherin and an increase in the abundance of the mesenchymal adhesion protein vimentin (Fig. 7), as previously shown with TGF $\beta 1$. We then explored whether erlotinib treatment was sufficient to activate IGF-1R at EMT-related sub-cellular localizations and whether pharmacological inhibition of IGF-1R could prevent erlotinibinduced EMT in PC-9 cells. The formation of marginal cellular 
protrusions that stained positively for the active form of IGF-1R at the tips of the F-actin stress fibers was clearly observable in response to graded concentrations of erlotinib (Supplementary Fig. 2, top panels); the EMT-related re-localization of phospho-active IGF-1R to EMT-related sub-cellular structures was fully preventable when treatment with erlotinib was performed in the presence of the IGF$1 \mathrm{R}$ inhibitor AG1024. Importantly, the drastic increase in the abundance of the mesenchymal adhesion protein vimentin induced in response to erlotinib cannot take place in the presence of the IGF-1R inhibitor AG1024 (Supplementary Fig. 2, top panels), thus substantiating that erlotinib-induced EMT-like features took place through activation of IGF-1R in a linear fashion.

We finally explored the impact of erlotinib treatment on the EMT phenotype in a NSCLC cell line panel with a well-known profile of erlotinib responsiveness and the mechanisms involved (Fig. 8). Similarly to EGFR-mutant PC-9 cells, the expression of E-cadherin was notably decreased or lost in the positive-control epidermal cell line, A431, which harbors wild-type EGFR gene amplification, in response to erlotinib. Unlike PC-9 cells, however, the undetectable level of the mesenchymal marker vimentin observed in untreated A431 cells remained unaltered following erlotinib treatment. EGFR wild-type $\mathrm{H} 460$ cells, an erlotinib-refractory mesenchymal cell line, notably failed to modulate the abundance of the mesenchymal adhesion protein vimentin in response to erlotinib. Immunofluorescence studies revealed that E-cadherin was associated with disorganized adhesive structures rather than restricted to the plasma membrane at areas of cell-cell contact in erlotinib-refractory H1975 cells, which express both the erlotinib-sensitizing L858R mutation and the $T 790 M$ resistant mutation. Interestingly, erlotinib treatment further enhanced the abnormal distribution of E-cadherin in various cytoplasmic locations including cell edges without adjacent cells and peri-nuclear areas. This lack of E-cadherin staining restricted to areas of cell-cell contact was accompanied by a conspicuous redefinition of cell shape and morphology, including a remarkable formation of long stress fibers of F-actin filaments; accordingly, basal expression of the mesenchymal marker vimentin was further enhanced in
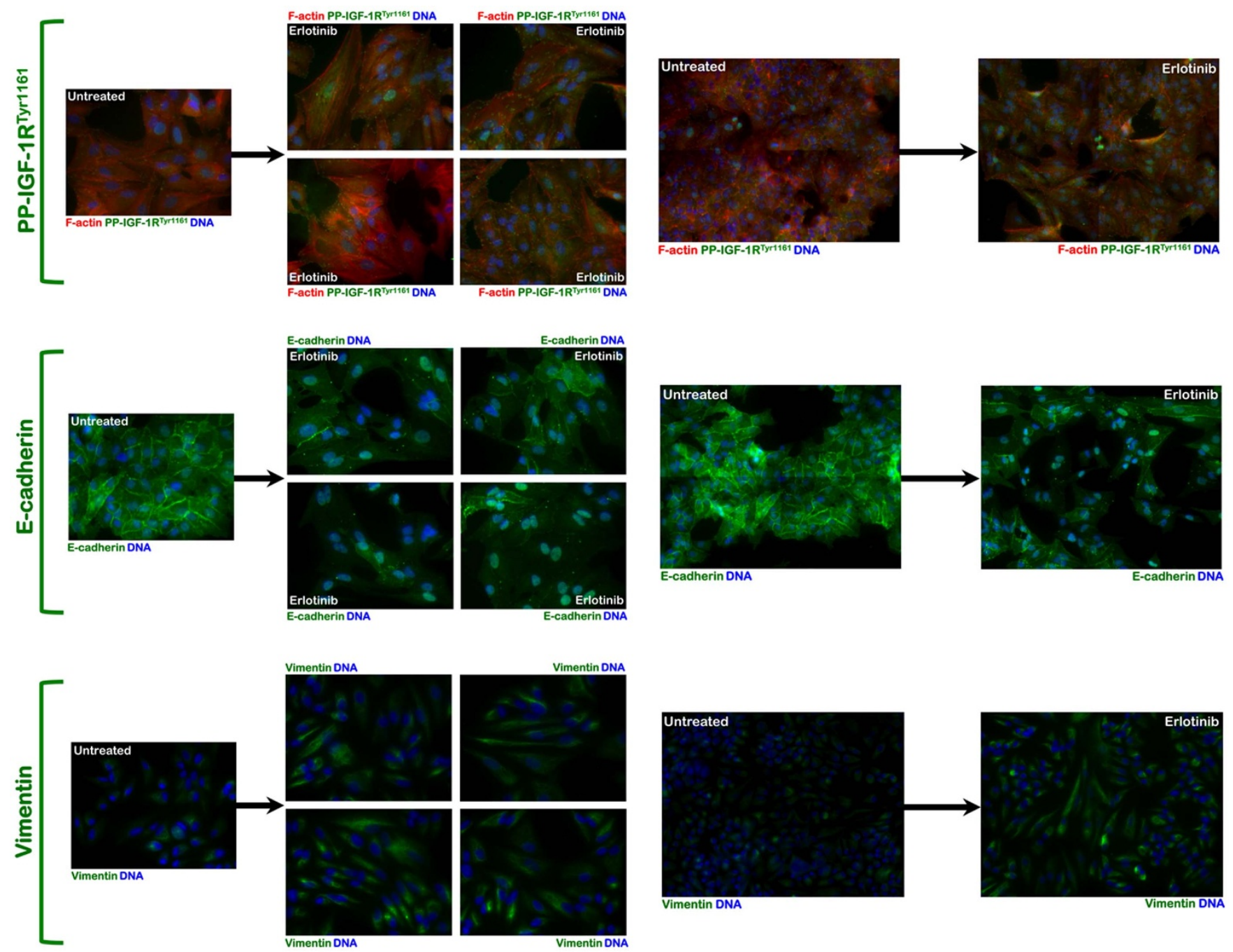

Figure 7 Short-term exposure to erlotinib activates EMT-like features in PC-9 cells. PC-9 cells were grown until 75\%-80\% confluence, serum-starved for $24 \mathrm{~h}$, and then treated for $72 \mathrm{~h}$ with $1 \mu \mathrm{mol} / \mathrm{L}$ erlotinib. Top panels. After fixation and permeabilization, cellular co-distribution of PP-IGF-1R $\mathrm{R}^{\mathrm{Ty} 1161}$ and F-actin was assessed following staining with anti-PP-IGF-1R ${ }^{\text {Tyr1161 }}$ and anti-F-actin antibodies, as specified, and Hoechst 33258 for nuclear counterstaining. Middle panels. After fixation and permeabilization, sub-cellular distribution of the epithelial marker E-cadherin was assessed following staining with an anti-E-cadherin antibody and Hoechst 33258 for nuclear counterstaining. Bottom panels. After fixation and permeabilization, subcellular distribution of the mesenchymal-specific marker vimentin was assessed following staining with an anti-vimentin antibody and Hoechst 33258 for nuclear counterstaining. Images show representative portions (left panels) or $4 \times 4$ montages (right panels) of untreated- or erlotinib-treated PC-9 cell cultures captured in different channels for PP-IGF-1R $\mathrm{R}^{\text {Tyr1161 }}$ (green), F-actin (red), E-cadherin (green), vimentin (green), and Hoechst 33258 (blue) with a $20 \times$ objective, and merged on BD Pathway 855 Bioimager System using BD Attovision software. 
erlotinib-treated H1975 cells. EGFR wild-type H1993 epithelial cells, which have an amplified MET allele that confers resistance to erlotinib, failed to alter the immunostaining pattern of the tight junctional protein E-cadherin at well-defined cell-cell adhesion complexes in response to treatment with erlotinib.

\section{Discussion}

NSCLC tumors carrying EGFR-activating mutations (e.g., exon 19 deletions or $L 858 R$ ) respond preferentially to the EGFR TKIs gefitinib and erlotinib. Despite a dramatic initial response to such inhibitors, over time (median of 6-12 months), most NSCLC patients develop drug resistance and relapse. Intense research efforts over the past few years have identified two major mechanisms of acquired resistance to gefitinib/erlotinib: secondary resistance mutations (e.g., T790M) and "oncogene kinase switch" systems (e.g., MET amplification and AXL activation). However, the precise mechanisms underlying acquired resistance in the $30-40 \%$ of EGFR TKI-resistant EGFR-mutated tumors that do not carry secondary resistance mutations or MET amplification remain largely unclear. Therefore, it is extremely important to continue to study preclinical models and/or tumors from NSCLC patients that have developed gefitinib/erlotinib resistance to uncover novel mechanisms of resistance to EGFR TKIs.

While our current pre-clinical model of delE746-A750-mutated PC-9 NSCLC cells with acquired resistance to erlotinib apparently lacked alterations in MET and AXL, our data initially confirmed that acquired resistance to erlotinib apparently developed through a "kinase switch" mechanism involving IGF-1R RTK hyperactivity. A noteworthy weakness of our current approach (i.e., erlotinib-resistant pooled populations were obtained from lung carcinoma cells originally harboring an erlotinib-sensitizing EGFR exon 19 deletion) is that pooled populations may contain cells with varying degrees of resistance, and the pools developed during this study may harbor resistance acquired over time or may represent a selected subpopulation of cells with inherent resistance. However, it should be acknowledged that erlotinib-refractory pooled populations better reflect the heterogeneous alterations that are naturally present in the majority of tumor cell populations, whereas clones, another possible approach, represent just one isolated alteration. Nonetheless, in our current EGFR T790M-, MET-, and AXL-independent scenario of acquired resistance to erlotinib, the EGFR signaling platform remained largely intact (i.e., EGFR protein levels remained unchanged, and the EGFR RTK activity remained active in the absence or presence of erlotinib), whereas IGF-1R appeared to assume a prominent role in regulating cell survival during the development of acquired erlotinib resistance. As a consequence, simultaneous inhibition of EGFR and IGF-1R activity becomes imperative to overcome erlotinib resistance. Indeed, the fact that the combination of erlotinib plus IGF-1R inhibitors (I-OMe-AG538 or AG1024) fully prevents the acquired erlotinib-resistant phenotype is consistent with IGF-1R playing a major role in this process. While preparing this manuscript, Cortot et al..$^{31}$ reported that PC-9-derived clones with acquired resistance to mutant-selective EGFR inhibitors and irreversible quinazoline EGFR inhibitors in the absence of EGFR T790M demonstrate a significantly activated IGF-1R signaling, and pharmacological inhibition of IGF-1R restored sensitivity to EGFR inhibitors.

Despite no patient data supporting the role of IGF-1R in acquired resistance to gefitinib/erlotinib, we are beginning to accumulate evidence from preclinical models indicating that clinical trials of IGF-1R inhibitors with demonstrated activity in unselected NSCLC patients might play a role in association with EGFR inhibitors in the management of NSCLCs with acquired resistance to gefitinib/erlotinib $^{32,33}$. It should be noted that increased IGF-1R activation in erlotinib-refractory delE746-A750-mutated PC-9 cells occurred in our lab models independently of changes in IGF-binding proteins (IGFBPs) (data not shown). Whereas earlier studies have demonstrated that a loss of IGFBP (IGFBP-3 and IGFBP-4) expression levels in tumor cells treated with EGFR TKIs activated IGF-1R signaling, which in turn mediated resistance to an EGFR antagonist ${ }^{20,31}$, our analyses failed to detect significant changes in the secretion levels of IGFBPs in delE746-A750-mutated PC-9 cells with acquired resistance to erlotinib. Instead, substantial evidence has related hyperactive IGF-1R signaling with a drastic enrichment in EMT-related morphological and transcriptional features. Our results suggested that a mesenchymal status-related activation of IGF-1R was
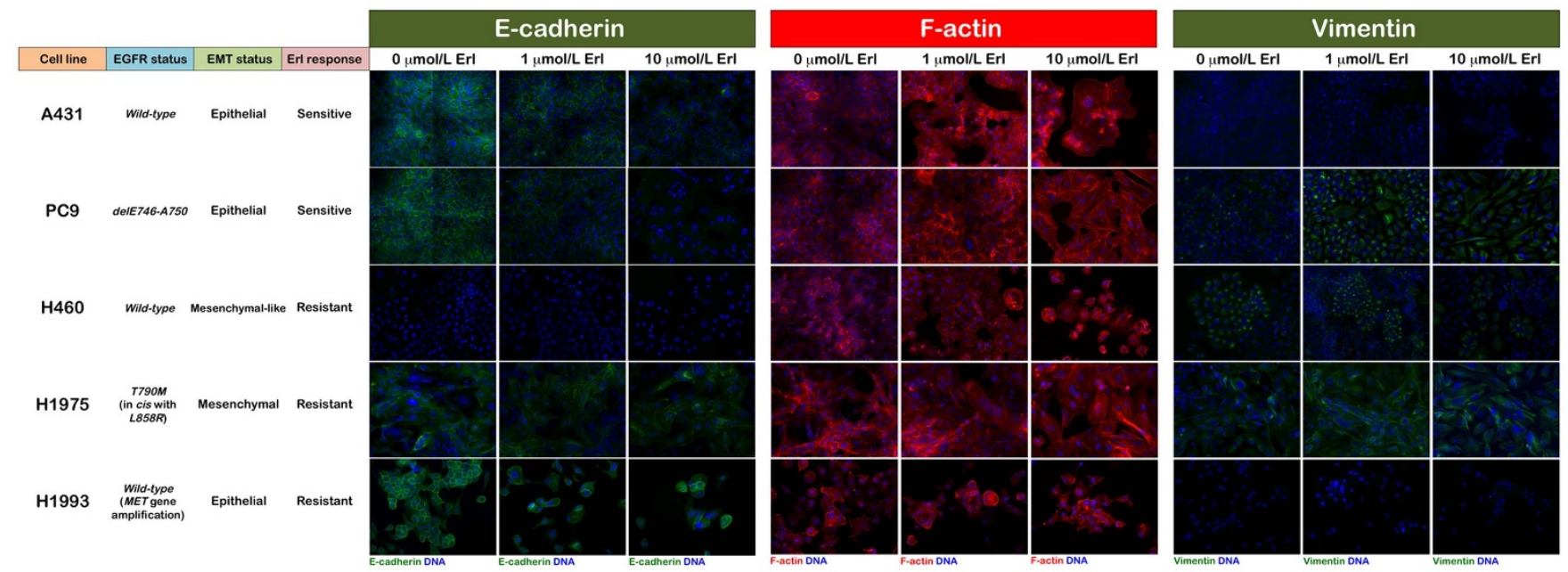

Figure 8 Effects of erlotinib treatment on the epithelial/mesenchymal phenotype of NSCLC cells. A431 (positive control), PC-9, H460, H1975, and H1993 cells were grown until 75\%-80\% confluence, serum-starved for $24 \mathrm{~h}$, and then treated for $72 \mathrm{~h}$ with graded concentrations ( 1 and $10 \mu$ mol/L) of erlotinib. Left panels. After fixation and permeabilization, sub-cellular distribution of the epithelial marker E-cadherin was assessed following staining with an anti-E-cadherin antibody and Hoechst 33258 for nuclear counterstaining. Middle panels. After fixation and permeabilization, sub-cellular distribution of F-actin was assessed following staining with an anti-F-actin antibody and Hoechst 33258 for nuclear counterstaining. Right panels. After fixation and permeabilization, sub-cellular distribution of the mesenchymal-specific marker vimentin was assessed following staining with an antivimentin antibody and Hoechst 33258 for nuclear counterstaining. Images show representative $4 \times 4$ montages of untreated- or erlotinib-treated cell cultures captured in different channels for E-cadherin (green), F-actin (red), vimentin (green), and Hoechst 33258 (blue) with a $20 \times$ objective, and merged on BD Pathway 855 Bioimager System using BD Attovision software. 
ultimately responsible for conferring resistance to erlotinib to delE746-A750-mutated PC9 cells. Indeed, our current study suggest for the first time that NSCLC cells' intrinsic plasticity to undergo an IGF-1R/EMT cross-talk in response to erlotinib can sufficiently abolish the erlotinib-sensitizing effect of highly prevalent EGFR mutations. On the one hand, the induction of an EMT phenomenon that desensitizes delE746-A750-mutated PC-9 cells to erlotinib was found to proceed, at least in part, through the activation of IGF-1R -i.e., TGF $\beta 1$-induced EMT is accompanied by IGF-1R activation at newly formed EMT-related sub-cellular structures and the EMT process triggered by TGF $\beta 1$ cannot fully occur in the presence of IGF-1R inhibitors. On the other hand, short-term exposure to erlotinib was able to potentiate the appearance of EMT-like features in delE746A750-mutated PC-9 cells, including stress fibers-associated activation of IGF-1R. Our data appear to support a previously unrecognized scenario in which, in the presence of erlotinibsensitizing EGFR mutations (delE746-A750, L858R), an aberrant/ incomplete epithelial differentiation might determine the intrinsic ability of EGFR mutant-NSCLC cells to rapidly shift toward the erlotinib-refractory mesenchymal end of the epithelial-mesenchymal spectrum. In our hands, acute treatment of EGFR-mutated PC-9 parental cells with high-dose erlotinib was found to notably activate an EMT-like phenomenon as part of the transcriptomic signature associated with erlotinib-induced cell growth inhibition. Moreover, the fact that erlotinib-enhanced cross-talk between IGF$1 \mathrm{R}$ and EMT signaling pathways was concomitant with a reduction in PC-9 cell numbers strongly suggests that erlotinib-induced cell death is not an obligatory outcome of erlotinib treatment in NSCLC cells harboring erlotinib-sensitizing EGFR exon 19 deletion mutations. The IGF-1R/EMT crosstalk appears to facilitate survival in erlotinib-naïve delE746-A750-mutated NSCLC cells, likely promoting their own resistance if erlotinib-induced blockade of EGFR persists. If the magnitude and/or duration of induced EMT may determine the fate of erlotinib-treated EGFR-mutated NSCLC cells (i.e., EMT is fully induced as a survival mode but fail to rescue erlotinib-sensitive cells), the intrinsic plasticity of EGFR-mutated cells to rapidly switch erlotinib-induced EMT as a tumor growthinhibitory mechanism to a mechanism of tumor survival may dictate the likelihood of the duration of erlotinib response. Upon chronic exposure to erlotinib, therefore, activation of the EMT genetic program appears to paradoxically switch from "growth inhibitory" to "pro-survival" in response to erlotinib-induced cell injuries. This molecular switch might be explained when considering the pre-existence of IGF-1R-positive EMT-like clones inherently resistant to a wide variety of tumoricidal insults including erlotinib-induced blockade of EGFR-driven tumor growth; in this scenario, it could be hypothesized that chronic exposure to erlotinib negatively select initially dominant epithelial-sensitive clones and promotes selection of mesenchymal-refractory tumor clones constitutively harboring an up-regulated IGF-1R and/or EMT signaling cascade. Alternatively, thresholds may exist within EGFR-mutated NSCLC cells for the magnitude of erlotinib-induced EMT that leads to cell death versus the magnitude of erlotinib-induced EMT that leads to cell survival and acquired resistance. Indeed, the main inducers of EMT (e.g., TGF $\beta$ ) can also promote apoptotic cell death, which suggest that EMT could potentially serve as a pathway to escape from cell death depending on the particular microenvironmental milieu. It should be noted that reduced proliferation has been suggested to be an integral part of the EMT-induced phenotype that is important for supporting survival of disseminated metastatic cells. Erlotinib-induced EMTrelated processes to reduce proliferation in favor of enhanced cell motility and survival, might provide an early selective advantage to EGFR-mutated NSCLC cells treated with erlotinib. Moreover, given that EMT can directly generate cancer stem cell cells by directly linking the acquisition of cellular motility with both the maintenance of tumor-initiating (stemness) capacity and the acquired multi-resistance to cancer therapeutics, early suppression of cellular proliferation by activation of an EMT genetic program may promote the selective activation of CSC cellular states to lastly generate erlotinib-refractory, CSC-enriched EGFR-mutated NSCLC cell populations. While further clonal studies are evidently required to unambiguously uncover the ultimate molecular pathways regulating the timing between early erlotinib-induced EMT with late EMTdriven resistance to erlotinib (e.g., it could be relevant to address whether erlotinib-induced non-viable cells show differential levels of IGF-1R activation and/or epithelial-mesenchymal features $a b$ initio), our findings may provide a putative mechanism underlying the emergence of CSC-like properties in EGFR-mutated NSCLC cells continuously exposed to high-concentrations of erlotinib.

Dual IGF-1R/EMT-targeting strategies may circumvent acquired resistance to erlotinib. Combined therapeutic inhibitions of EGFR and IGF-1R to clinically abrogate this acquired mechanism of drug resistance in EGFR-mutated NSCLC patients refractory to erlotinibbased treatments might require the use of EMT markers to ensure a more responsive patient population prior to the initiation of subsequent lines of therapy. In this regard, it is noteworthy that molecular analysis of NSCLC responses to IGF-1R inhibition revealed that intrinsic sensitivity to figitumumab, a monoclonal antibody against IGF-1R, was significantly related to the simultaneous occurrence of markers of the IGF-1R pathway in the EMT pathway ${ }^{34}$. Although it remains unclear whether high-levels of IGF-1R expression are directly associated with high IGF-1R kinase activity levels, IGF-1R is among a small group of oncogenes that can confer in vivo tumorigenicity when overexpressed alone via IGFR-1R-mediated induction of the transcriptional repressor SNAIL and downregulation of E-cadherin ${ }^{35}$. Indeed, recent results from our laboratory have confirmed that regardless of erlotinib treatment, EMT-enriched erlotinib-resistant PC-9 tumor xenografts exhibited a significantly enhanced tumor engraftment and faster growth compared with erlotinib-sensitive PC-9 parental cells (data not shown). It could be argued that coupling IGF-1R activation with well-recognized EMT abilities, such as migration, invasiveness, and metastasis, cannot explain the enhanced cell survival of EGFR-mutated NSCLC cells challenged with erlotinib. However, we should acknowledge that apart from their direct implication in the EMT phenomenon, EMT transcriptional drivers (e.g., SNAIL factors) can also regulate other cellular processes related to cell proliferation and survival, thus contributing to resistance to pro-apoptotic stresses ${ }^{36-39}$. Accordingly, we recently revealed that a significant reduction in tumor growth and dramatic gain in sensitivity to the growth-inhibitory effects of the anti-HER2 monoclonal antibody trastuzumab in vivo can be observed upon injection of SLUG knocked-down cells into nude mice ${ }^{40}$. The promotion of cell survival as a primary function of EMT factors such as SLUG, which can rescue cells from apoptosis by repressing pro-apoptotic factors that otherwise would promote cell death during a partial EMT, is strongly supported by the fact that exogenous addition of the EMT inducer TGF $\beta 1$, which efficiently promoted a transient and reversible trans-differentiation of delE746A750-mutated cells to mesenchymal-like phenotypes, was sufficient to significantly induce refractoriness to the cytotoxic effects of erlotinib. In this regard, it should be noted that E-cadherin appeared to associate with disorganized adhesive structures in delE746-A750mutated PC-9 epithelial cells. Unlike bona fide epithelial cells in which the localization of E-cadherin is restricted to the plasma membrane at areas of cell-cell contact, E-cadherin was found across the entire surface of the plasma membrane and was notably distributed in various cytoplasmic localizations in EGFR-mutated PC-9 cells. Indeed, E-cadherin staining was notably diffused at areas of cell-cell contact, and PC-9 cells normally exhibited reduced and punctuated E-cadherin staining that was not restricted to areas of cell-cell contact but was also observed along cell edges without adjacent cells. These findings may suggest that EGFR-mutated NSCLC cells can exhibit a 
"mixed phenotype" in which E-cadherin is included in disorganized adhesive structures that largely lack junctional organization and, therefore, they might be prompted to rapidly suffer erlotinib-desensitizing EMT phenomena in response to certain stimuli including erlotinib treatment itself. These findings, together with the fact that exogenous TGF $\beta 1$ and erlotinib notably cooperate to promote the acquisition of mesenchymal cell features in erlotinib-naïve PC-9 cell cultures and the exquisite ability of erlotinib-refractory PC-9/ErlR pooled populations to further enhance their EMT-associated features in response to TGF $\beta 1$ (data not shown), strongly suggest that crosstalk between TGF $\beta 1$ and IGF-1R signaling pathways might uncouple EGFR activity from erlotinib functioning by activating the EMT phenomenon. Using erlotinib hyper-resistant cells established from H1650 cells, which are EGFR mutant but unresponsive to erlotinib due to PTEN deletion, Yao et $\mathrm{al}^{41}$ were pioneers at uncovering the existence of a TGF $\beta$-driven subpopulation of NSCLC cells intrinsically resistant to erlotinib that display features suggestive of EMT. Using the erlotinib-sensitive EGFR mutant NSCLC cell line HCC4006 harboring the delL747-E749, A750P mutation in EGFR exon 19, Suda et al. ${ }^{29}$ established that mesenchymal status, not a specific oncogenic-activated protein, could sufficiently promote loss of EGFR dependence to confer resistance to erlotinib. More recently, Chang et al. ${ }^{42}$ confirmed that in the absence of the common secondsite EGFR mutation T790M or MET amplification, acquired resistance to gefitinib occurs in EGFR-mutated PC9 cells that have undergone EMT. In agreement with the above-mentioned pro-survival role of EMT TFs, the authors observed that SLUG overexpression efficiently protected PC9 cells from gefitinib-induced apoptosis; accordingly, specific knockdown of SLUG can reverse secondary resistance to gefitinib. Importantly, SLUG expression in clinical samples was significantly higher after the development of acquired resistance to EGFR TKIs than in treatment-naïve samples ${ }^{42}$.

Earlier studies have suggested an active involvement of EMT in resistance to EGFR TKIs ${ }^{25,27,43,44}$, and accordingly, E-cadherin expression has been reported to operate as a biomarker capable of predicting the clinical efficacy of gefitinib ${ }^{27}$. However, it should be noted that these studies were largely based on NSCLC cells expressing wild-type EGFR; therefore, these studies did not mimic clinical usage of EGFR TKIs because EGFR wild-type NSCLC cells are intrinsically resistant to EGFR TKIs. We have now confirmed that acquired resistance to erlotinib in NSCLCs harboring erlotinib-sensitizing EGFR mutations could be mediated by EMT induction through transcriptional reprogramming; the sole activation of a minimal EMT-mRNA/protein signature, including upregulation of the master EMT gene SLUG and suppression of E-cadherin, appeared to be informative of a significantly reduced responsiveness to erlotinib, even if transiently activated. In this scenario, it might appear reasonable to suggest that in addition to the presence of the T790M mutation, immunohistochemical and/or microarray-based transcriptional profiling studies dedicated to specific alterations of EMT drivers (e.g., SLUG) and/or effectors (e.g., vimentin) may be helpful in monitoring for erlotinib responders and non-responders based on the loss of biomarkers associated with epithelial status and gain of biomarkers associated with mesenchymal status. A microRNA gene expression signature targeting EMT has been recently shown to predict response to erlotinib ${ }^{45}$. Byers et al. ${ }^{46}$ recently developed a robust EMT signature that highlights different drug responsiveness patterns for epithelial and mesenchymal NSCLC cells and can predict resistance to EGFR TKIs regardless of EGFR mutation status. It might be tempting to suggest that such "mesenchymal transition signatures" could be employed as predictive signatures that may merit validation in samples from both completed and prospective erlotinib-based clinical trials. Erlotinib therapy-based clinical trials might be designed with predictive EMT-related marker validation in mind following a rigorous design because although it is plausible that not all EMT markers included in a "mesenchymal transition signature" will meet all surrogacy criteria, their use could provide crucial information on the occurrence of acquired resistance to erlotinib. We should acknowledge, however, that a "mesenchymal transition signature" likely reflects a reversible process in which the mesenchymal markers may dynamically appear and disappear depending on intrinsic (i.e., cell autonomous) and/or microenvironmental signals ${ }^{41}$. Consequently, the potential use of EMT-like predictive signatures would have high selectively but not might have the sensitivity to predict acquired resistance to erlotinib. The presence of the EMT signature in EGFR-mutant tumors would likely imply inherent or acquired resistance to erlotinib; however, given the heterogeneity in the mechanisms of acquired resistance, the lack of the signature in a particular sample from an EGFR mutantNSCLC might not indicate erlotinib responsiveness because the signature may have appeared earlier.

The clinical implications of our data are that erlotinib efficacy in EGFR-mutant NSCLC patients that have acquired resistance to EGFR TKIs such as erlotinib might be restored with therapies that block or reverse the IGF-1R/EMT crosstalk. Specific targeted therapies directed against the cell survival pathways (e.g., IGF-1R) of mesenchymal-like cells would be predicted to reduce the progression of EGFR-mutant NSCLCs on erlotinib. In this regard, it is worth noting that a landmark study showing that the addition of silibinin, which is the active constituent of silymarin isolated from the dried fruits of milk thistle (Silybum marianum) plant ${ }^{47-50}$, to EGFR TKIs drastically suppresses tumor growth in primary and acquired-resistance cells with the EGFR T790M mutation ${ }^{51}$ may open a new therapeutic avenue to clinically manage EMT-driven acquired resistance to erlotinib. Silibinin appears to operate as a potent natural agent leading to the reversal of EMT by decreasing the levels of key EMT TFs (e.g., SLUG) and increasing the expression levels of E-cadherin $^{52-54}$. Silibinin has been shown to reduce IGF-1R phosphorylation, indicating an inhibitory effect on the IGF-1R-mediated signaling pathway in cancer cells ${ }^{55,56}$. Through using a milk thistle extract rich in silybin meglumine, a commercially used water-soluble form of silibinin ${ }^{57}$, we are currently testing whether the anti-IGF-1R/ EMT activity of silibinin can translate into the reversal of acquired resistance to EGFR TKIs in animal models. A dual targeting of IGF$1 \mathrm{R}$ and EMT might be crucial to prevent and/or circumvent acquired resistance to erlotinib in EGFR-mutated cells because, although early studies by Morgillo et al. ${ }^{58}$ provided evidence that erlotinib induces heterodimerization of EGFR/IGF-1R, we failed to observe either colocalization of the phospho-active forms of EGFR and IGF-1R or changes in the phosphorylation status of EGFR upon pharmacological blockade of IGF-1R in EMT-like, erlotinib-refractory pooled populations (data not shown). Positive anti-IGF-1R/EMT findings may warrant further clinical evaluation of silibinin derivatives to effectively delay or prevent the development of acquired resistance to erlotinib in EGFR-mutant NSCLC patients.

During the preparation of this manuscript, Shien et al..$^{59}$ reported that acquired resistance to the EGFR inhibitor gefitinib was associated with a manifestation of stem cell-like properties in EGFRmutated NSCLC cells, including EMT features, overexpression of the stem cell-marker aldehyde dehydrogenase (ALDH), increase of side-population, and self-renewal capacity. Recent findings in our laboratory have confirmed that erlotinib-refractory PC-9 derivatives in which hyperactive IGF-1R signaling associates with enrichment in EMT-related morphological and transcriptional features are similarly enriched with stem-cell like properties and contain drastically higher percentages $(>4,000 \%)$ of $\mathrm{ALDH}^{\text {bright }}$ cells than erlotinibresponsive parental cells (unpublished observations). While the majority of previously reported cells that acquired resistance to the EGFR TKIs gefitinib and erlotinib were established with stepwise escalation of the EGFR TKI concentration, when Shien et al. ${ }^{59}$ established gefitinib-resistant cells with both the stepwise escalation method and the high-concentration exposure method, they observed 
that the manner of exposure to the EGFR TKI notable influenced the mechanism of acquired resistance; in their hands, the emergence of CSC-like properties solely occurred in response to high-concentration exposure but not following exposure to very low EGFR TKI concentrations contributing to the step-wise selection of resistant cells. It is noteworthy that the high-concentration exposure method similarly induced EMT features and enrichment of cells with CSClike properties in our PC-9 model of acquired resistance to erlotinib. If cells intrinsically enriched with EMT/CSC-like properties may exist as minor clones in heterogenous EGFR-mutated NSCLC cell populations, and they are finally selected as the major clones, both selection methods would be expected to similarly enrich with EMT/ CSC-like properties the population acquiring resistance to EGFR TKIs. Although future studies should be designed to unambiguously clarify whether the high-concentration exposure directly induces the emergence of EMT/CSC-like properties to mimic a clinically relevant event, we should acknowledge that our ability to delineate novel features of resistance to EGFR TKIs in EGFR-mutated NSCLC might significantly depend on the experimental procedure chosen to select resistant populations in vitro.

\section{Methods}

Cell line. PC-9 NSCLC-derived cells expressing the EGFR exon 19 deletion mutation (delE746-A750) were obtained from the IBL cell bank (Gunma, Japan). A431, H460, H1975, and H1993 cell lines were obtained from the American Type Culture Collection (Manassas, VA, USA).

Generation of PC-9 cells with acquired resistance to erlotinib. To establish EGFRmutant NSCLC cells with acquired resistance to erlotinib, cultures of PC-9 NSCLC cells were continuously exposed to high doses of erlotinib $(1 \mu \mathrm{mol} / \mathrm{L})$ in routine culture medium, which was replaced every one to two days for approximately two months. Initially, PC- 9 cells numbers were substantially reduced, and for the next 3 months, the surviving cells were passaged in the presence of up to $10 \mu \mathrm{mol} / \mathrm{L}$ erlotinib approximately every $10-12$ days, with a seeding ratio of $1: 2$. The cell proliferation rates slowly increased over the next two months to allow passaging every 5-7 days, with a seeding ratio of $1: 5$. A stable growth rate was reached after a total of 6 months with routine maintenance of the PC-9/ErlR (erlotinib-resistant) pooled populations involving passages every 5 days, with a seeding ratio of $1: 10$ of the confluent cell culture grown in the presence of $10 \mu \mathrm{mol} / \mathrm{L}$ erlotinib.

Drugs and chemicals. The EGFR (HER1) TKI erlotinib (Tarceva ${ }^{\circledR}$ ) was a kind gift from Roche Pharmaceuticals. Stock solutions of erlotinib at $10 \mathrm{mmol} / \mathrm{L}$ were prepared in DMSO and stored in aliquots in the dark at $-20^{\circ} \mathrm{C}$ until utilization. Recombinant human (carrier-free) TGF- $\beta 1$ was purchased from R\&D Systems Inc. (Minneapolis, MA). IGF-1R inhibitors I-Ome-AG538 and AG1024 were obtained from EMD Calbiochem (La Jolla, CA) and dissolved in DMSO at a stock concentration of $10 \mathrm{mmol} / \mathrm{L}$. For experimental use, all drugs solutions were prepared fresh from stock solutions and were diluted with cell growth medium. Control cells were cultured in media containing identical concentrations $(v / v)$ of vehicle.

Phosphoproteome profiling. Erlotinib-sensitive PC-9 parental cells and erlotinibrefractory PC-9 pooled populations were plated in $100-\mathrm{mm}$ tissue culture dishes and cultured in regular media containing $10 \%$ FBS until they reached $75-80 \%$ confluence. The cells were then washed twice with serum-free medium and incubated overnight upon serum-free conditions. Cells were then stimulated to grow in 5\% FBScontaining medium in the presence or absence of erlotinib $(1 \mu \mathrm{mol} / \mathrm{L}$ for PC-9 parental cells and $10 \mu \mathrm{mol} / \mathrm{L}$ for PC-9 POOLs) for $48 \mathrm{~h}$. Cells were rinsed with coldPBS and immediately solubilized in NP-40 lysis buffer [1\% NP-40, $20 \mathrm{mmol} / \mathrm{L}$ Tris$\mathrm{HCl}$ (pH 8.0), $137 \mathrm{mmol} / \mathrm{L} \mathrm{NaCl}, 10 \%$ glycerol, $2 \mathrm{mmol} / \mathrm{L}$ EDTA, $1 \mathrm{mmol} / \mathrm{L}$ sodium orthovanadate, $10 \mu \mathrm{g} / \mathrm{mL}$ aprotinin, $10 \mu \mathrm{g} / \mathrm{mL}$ leupeptin] by rocking the lysates gently at $4{ }^{\circ} \mathrm{C}$ for $30 \mathrm{~min}$. Following microcentrifugation at $14,000 \times \mathrm{g}$ for $5 \mathrm{~min}$, supernatants were transferred into a clean test tube and sample protein concentrations were determined using the Pierce Protein assay kit (Rockford, IL). Lysates were diluted and incubated with Human Phospho-RTK Arrays (Proteome Profiler; R\&D Systems; Minneapolis, MN, USA) as per the manufacturer's instructions. In this method, capture and control antibodies have been spotted in duplicate on nitrocellulose membranes. Briefly, the membranes were blocked with $5 \%$ bovine serum album (BSA)/TBS [0.01 mol/L Tris $\mathrm{HCl}, \mathrm{pH}$ 7.6] for $1 \mathrm{~h}$. Membranes were then incubated with $750 \mu \mathrm{g}$ of total protein. After extensive washing with TBS including $0.1 \% v / v$ Tween-20, 3 times for $5 \mathrm{~min}$, to remove unbound materials, the membranes were then incubated with HRP-phospho-RTK antibodies for $2 \mathrm{~h}$ at room temperature. Unbound HRP antibody was washed out with TBS including $0.1 \% \mathrm{v} / \mathrm{v}$ Tween-20. Finally, array data were developed on X-ray film using a chemiluminescence detection system (Amersham Life Sciences).

Cell viability assays. The effect of erlotinib on the cell viability was determined using a standard colorimetric MTT (3-4, 5-dimethylthiazol-2-yl-2, 5-diphenyl-tetrazolium bromide) reduction assay. For each treatment, the cell viability was evaluated as a percentage using the following equation: $\left(\mathrm{OD}_{570}\right.$ of the treated sample/OD $\mathrm{D}_{570}$ of the untreated sample) $\times 100$.

Quantitative real-time polymerase chain reaction (qRT-PCR). The total RNA was extracted from cell cultures using a Qiagen RNeasy kit and QIAshredder columns according to the manufacturer's instructions. One microgram of total RNA was reverse-transcribed to cDNA using the Reaction Ready ${ }^{\mathrm{TM}}$ First Strand cDNA Synthesis Kit (SABiosciences) and applied to a customized PCR array (96-well format) containing the following panel of genes: GSC, KRT14, KRT19, NUMB, TCF3, TCF4, SDC1, ZO-1, CD44, TWIST, SNAI1, VIM, SLUG, CDH1, ZEB1, CDH2, ZEB2, $F N 1$, and CD24. The arrays were processed according to the SABiosciences RT-PCR manual and analyzed using an Applied Biosystems 7500 Fast Real-Time PCR System with an automated baseline and threshold cycle detection. The data were interpreted using SABiosciences's web-based PCR array analysis tool.

Immunofluorescence staining and high-content confocal imaging. Cells were seeded at approximately 5,000 cells/well in 96-well clear-bottom imaging tissue culture plates (Becton Dickinson Biosciences; San Jose, CA) optimized for automated imaging applications. Triton ${ }^{\circledR}$ X-100 permeabilization and blocking, primary antibody staining, secondary antibody staining using Alexa Fluor ${ }^{\circledR} 488 / 594$ goat antirabbit/mouse IgGs (Invitrogen, Probes, Eugene, Oregon) and counterstaining (using Hoechst 33258; Invitrogen) were performed following the BD Biosciences protocols. The images were captured in different channels for Alexa Fluor ${ }^{\circledR} 488$ (pseudocolored green), Alexa Fluor ${ }^{\circledR} 594$ (pseudocolored red), and Hoechst 33258 (pseudocolored blue) using a BD Pathway ${ }^{\mathrm{TM}} 855$ Bioimager System (Becton Dickinson Biosciences, San Jose, California) with $20 \times$ or $40 \times$ objectives (NA 075 Olympus). Merged images were obtained according to the Recommended Assay Procedure using the BD Attovision ${ }^{\mathrm{TM}}$ software. We employed the following antibodies: 1:50 dilution of antiPP-IGF-1R (Tyr 1161) (sc-101703; Santa Cruz Biotechnology, Santa Cruz, CA, USA), $1: 40$ dilution of Alexa Fluor 594 Phalloidin (A12381; Invitrogen), $1: 400$ dilution of anti-vinculin (V9264, clone hVIN-1, Sigma-Aldrich, St. Louis, MO, USA), and 1:200 dilution of anti-E-Cadherin (24E10) Rabbit mAb \#3195 (Cell Signaling Technology, Inc., Danvers, MA, USA)

Colony formation. The anchorage-dependent clonogenic growth assay was done by initially seeding PC-9 parental cells and erlotinib-refractory PC-9 derivatives into 100 -mm plates at low density (approximately $3 \times 10^{3}$ cells per well). Cells were either left untreated or incubated for 72 hours with different concentrations of erlotinib, IOme-AG538, AG1024 or combinations of the two drugs in serum-free RPMI medium in the presence of IGF $(50 \mathrm{ng} / \mathrm{mL})$. Cells were replated in six-well plates and cultured in drug-free medium for 7 to 10 days, in a humidified atmosphere with $5 \%$ $\mathrm{CO}_{2}$, at $37^{\circ} \mathrm{C}$, and then colonies were stained with crystal violet. We then counted the number of colonies with $>50$ cells.

Statistics. Two-group comparisons were performed by the Student $t$ test for paired and unpaired values. Comparisons of means of $\geq 3$ groups were performed by ANOVA, and the existence of individual differences, in case of significant $F$ values at ANOVA, tested by Scheffé's multiple comparisons. In all the cases statistical analyses were carried out with XLSTAT (Addinsoft ${ }^{\mathrm{TM}}$ ) and $\mathrm{P}<0.01$ was considered to be significant.

1. Lynch, T. J. et al. Activating mutations in the epidermal growth factor receptor underlying responsiveness of non-small-cell lung cancer to gefitinib. NEngl JMed 350, 2129-39 (2004).

2. Paez, J. G. et al. EGFR mutations in lung cancer: correlation with clinical response to gefitinib therapy. Science 304, 1497-500 (2004).

3. Pao, W. et al. EGF receptor gene mutations are common in lung cancers from "never smokers" and are associated with sensitivity of tumors to gefitinib and erlotinib. Proc Natl Acad Sci U S A 101, 13306-11 (2004).

4. Mitsudomi, T. \& Yatabe, Y. Mutations of the epidermal growth factor receptor gene and related genes as determinants of epidermal growth factor receptor tyrosine kinase inhibitors sensitivity in lung cancer. Cancer Sci 98, 1817-24 (2007).

5. Riely, G. J., Politi, K. A., Miller, V. A. \& Pao, W. Update on epidermal growth factor receptor mutations in non-small cell lung cancer. Clin Cancer Res 12, 7232-41 (2006).

6. Rosell, R. et al. Screening for epidermal growth factor receptor mutations in lung cancer. N Engl J Med 361, 958-67 (2009).

7. Riely, G. J. et al. Clinical course of patients with non-small cell lung cancer and epidermal growth factor receptor exon 19 and exon 21 mutations treated with gefitinib or erlotinib. Clin Cancer Res 12, 839-44 (2006)

8. Jackman, D. M. et al. Exon 19 deletion mutations of epidermal growth factor receptor are associated with prolonged survival in non-small cell lung cancer patients treated with gefitinib or erlotinib. Clin Cancer Res 12, 3908-14 (2006).

9. Kobayashi, S. et al. EGFR mutation and resistance of non-small-cell lung cancer to gefitinib. N Engl J Med 352, 786-92 (2005).

10. Pao, W. et al. Acquired resistance of lung adenocarcinomas to gefitinib or erlotinib is associated with a second mutation in the EGFR kinase domain. PLoS Med 2, e73 (2005). 
11. Kwak, E. L. et al. Irreversible inhibitors of the EGF receptor may circumvent acquired resistance to gefitinib. Proc Natl Acad Sci U S A 102, 7665-70 (2005)

12. Balak, M. N. et al. Novel D761Y and common secondary T790M mutations in epidermal growth factor receptor-mutant lung adenocarcinomas with acquired resistance to kinase inhibitors. Clin Cancer Res 12, 6494-501 (2006).

13. Kosaka, T. et al. Analysis of epidermal growth factor receptor gene mutation in patients with non-small cell lung cancer and acquired resistance to gefitinib. Clin Cancer Res 12, 5764-9 (2006).

14. Morgillo, F., Bareschino, M. A., Bianco, R., Tortora, G. \& Ciardiello, F. Primary and acquired resistance to anti-EGFR targeted drugs in cancer therapy. Differentiation 75, 788-99 (2007).

15. Nguyen, K. S., Kobayashi, S. \& Costa, D. B. Acquired resistance to epidermal growth factor receptor tyrosine kinase inhibitors in non-small-cell lung cancers dependent on the epidermal growth factor receptor pathway. Clin Lung Cancer 10, 281-9 (2009).

16. Lin, L. \& Bivona, T. G. Mechanisms of Resistance to Epidermal Growth Factor Receptor Inhibitors and Novel Therapeutic Strategies to Overcome Resistance in NSCLC Patients. Chemother Res Pract 2012, 817297 (2012).

17. Engelman, J. A. et al. MET amplification leads to gefitinib resistance in lung cancer by activating ERBB3 signaling. Science 316, 1039-1043 (2007).

18. Bean, J. et al. MET amplification occurs with or without T790M mutations in EGFR mutant lung tumors with acquired resistance to gefitinib or erlotinib. Proc Natl Acad Sci U S A 104, 20932-7 (2007).

19. Suda, K. et al. Reciprocal and complementary role of MET amplification and EGFR T790M mutation in acquired resistance to kinase inhibitors in lung cancer. Clin Cancer Res 16, 5489-98 (2010).

20. Guix, M. et al. Acquired resistance to EGFR tyrosine kinase inhibitors in cancer cells is mediated by loss of IGF-binding proteins. J Clin Invest 118, 2609-19 (2008).

21. Kokubo, Y. et al. Reduction of PTEN protein and loss of epidermal growth factor receptor gene mutation in Lung cancer with natural resistance to gefitinib (IRESSA). Br J Cancer 92, 1711-9 (2005).

22. Yamasaki, F. et al. Acquired resistance to erlotinib in A-431 epidermoid cancer cells requires down-regulation of MMAC1/PTEN and up-regulation of phosphorylated Akt. Cancer Res 67, 5779-88 (2007).

23. Zhang, Z. et al. Activation of the AXL kinase causes resistance to EGFR-targeted therapy in lung cancer. Nat Genet 44, 852-60 (2012).

24. Sequist, L. V. et al. Genotypic and histological evolution of lung cancers acquiring resistance to EGFR inhibitors. Sci Transl Med 3, 75ra26 (2011).

25. Thomson, S. et al. Epithelial to mesenchymal transition is a determinant of sensitivity of non-small-cell lung carcinoma cell lines and xenografts to epidermal growth factor receptor inhibition. Cancer Res 65, 9455-62 (2005).

26. Fuchs, B. C. et al. Epithelial-to-mesenchymal transition and integrin-linked kinase mediate sensitivity to epidermal growth factor receptor inhibition in human hepatoma cells. Cancer Res 68, 2391-9 (2008).

27. Yauch, R. L. et al. Epithelial versus mesenchymal phenotype determines in vitro sensitivity and predicts clinical activity of erlotinib in lung cancer patients. Clin Cancer Res 11, 8686-98 (2005).

28. Coldren, C. D. et al. Baseline gene expression predicts sensitivity to gefitinib in non-small cell lung cancer cell lines. Mol Cancer Res 4, 521-8 (2006).

29. Suda, K. et al. Epithelial to mesenchymal transition in an epidermal growth factor receptor-mutant lung cancer cell line with acquired resistance to erlotinib. J Thorac Oncol 6, 1152-61 (2011).

30. Chung, J. H. et al. Clinical and molecular evidences of epithelial to mesenchymal transition in acquired resistance to EGFR-TKIs. Lung Cancer 73, 176-82 (2011).

31. Cortot, A. B. et al. Resistance to Irreversible EGFR Tyrosine Kinase Inhibitors through a Multistep Mechanism Involving the IGF1R Pathway. Cancer Res 73, 834-43 (2013).

32. Karp, D. D. et al. Phase II study of the anti-insulin-like growth factor type 1 receptor antibody CP-751,871 in combination with paclitaxel and carboplatin in previously untreated, locally advanced, or metastatic non-small-cell lung cancer. J Clin Oncol 27, 2516-22 (2009).

33. Gualberto, A. \& Pollak, M. Emerging role of insulin-like growth factor receptor inhibitors in oncology: early clinical trial results and future directions. Oncogene 28, 3009-21 (2009).

34. Gualberto, A. et al. Molecular analysis of non-small cell lung cancer identifies subsets with different sensitivity to insulin-like growth factor I receptor inhibition. Clin Cancer Res 16, 4654-65 (2010).

35. Kim, H. J. et al. Constitutively active type I insulin-like growth factor receptor causes transformation and xenograft growth of immortalized mammary epithelial cells and is accompanied by an epithelial-to-mesenchymal transition mediated by NF-kappaB and snail. Mol Cell Biol 27, 3165-75 (2007).

36. Inoue, A. et al. Slug, a highly conserved zinc finger transcriptional repressor, protects hematopoietic progenitor cells from radiation-induced apoptosis in vivo. Cancer Cell 2, 279-88 (2002).

37. Kajita, M., McClinic, K. N. \& Wade, P. A. Aberrant expression of the transcription factors Snail and Slug alters the response to genotoxic stress. Mol Cell Biol 24, 7559-66 (2004).

38. Wu, W. S. et al. Slug antagonizes p53-mediated apoptosis of hematopoietic progenitors by repressing puma. Cell 123, 641-53 (2005).
39. Leroy, P. \& Mostov, K. E. Slug is required for cell survival during partial epithelial-mesenchymal transition of HGF-induced tubulogenesis. Mol Biol Cell 18, 1943-52 (2007)

40. Oliveras-Ferraros, C. et al. Epithelial-to-mesenchymal transition (EMT) confers primary resistance to trastuzumab (Herceptin). Cell Cycle 11, 4020-32 (2012).

41. Yao, Z. et al. TGF-beta IL-6 axis mediates selective and adaptive mechanisms of resistance to molecular targeted therapy in lung cancer. Proc Natl Acad Sci U S A 107, 15535-40 (2010)

42. Chang, T. H. et al. Slug confers resistance to the epidermal growth factor receptor tyrosine kinase inhibitor. Am J Respir Crit Care Med 183, 1071-9 (2011).

43. Frederick, B. A. et al. Epithelial to mesenchymal transition predicts gefitinib resistance in cell lines of head and neck squamous cell carcinoma and non-small cell lung carcinoma. Mol Cancer Ther 6, 1683-91 (2007).

44. Witta, S. E. et al. Restoring E-cadherin expression increases sensitivity to epidermal growth factor receptor inhibitors in lung cancer cell lines. Cancer Res 66, 944-5 (2006)

45. Bryant, J. L. et al. A microRNA gene expression signature predicts response to erlotinib in epithelial cancer cell lines and targets EMT. Br J Cancer 106, 148-56 (2012).

46. Byers, L. A. et al. An epithelial-mesenchymal transition (EMT) gene signature predicts resistance to EGFR and PI3K inhibitors and identifies Axl as a therapeutic target for overcoming EGFR inhibitor resistance. Clin Cancer Res 19, 279-290 (2013).

47. Post-White, J., Ladas, E. J. \& Kelly, K. M. Advances in the use of milk thistle (Silybum marianum). Integr Cancer Ther 6, 104-9 (2007).

48. Gazák, R., Walterová, D. \& Kren, V. Silybin and silymarin--new and emerging applications in medicine. Curr Med Chem 14, 315-38 (2007).

49. Agarwal, R., Agarwal, C., Ichikawa, H., Singh, R. P. \& Agarwal, B. B. Anticancer potential of silymarin: from bench to bed side. Anticancer Res 26, 4457-98 (2006).

50. Singh, R. P. \& Agarwal, R. Prostate cancer chemoprevention by silibinin: bench to bedside. Mol Carcinog 45, 436-42 (2006).

51. Rho, J. K. et al. Combined treatment with silibinin and epidermal growth factor receptor tyrosine kinase inhibitors overcomes drug resistance caused by T790M mutation. Mol Cancer Ther 9, 3233-43 (2010).

52. Singh, R. P., Raina, K., Sharma, G. \& Agarwal, R. Silibinin inhibits established prostate tumor growth, progression, invasion, and metastasis and suppresses tumor angiogenesis and epithelial-mesenchymal transition in transgenic adenocarcinoma of the mouse prostate model mice. Clin Cancer Res 14, 7773-80 (2008).

53. Wu, K. et al. Silibinin reverses epithelial-to-mesenchymal transition in metastatic prostate cancer cells by targeting transcription factors. Oncol Rep 23, 1545-52 (2010).

54. Deep, G., Gangar, S. C., Agarwal, C. \& Agarwal, R. Role of E-cadherin in antimigratory and antiinvasive efficacy of silibinin in prostate cancer cells. Cancer Prev Res (Phila) 4, 1222-32 (2011).

55. Zi, X., Zhang, J., Agarwal, R. \& Pollak, M. Silibinin up-regulates insulin-like growth factor-binding protein 3 expression and inhibits proliferation of androgen-independent prostate cancer cells. Cancer Res 60, 5617-20 (2000).

56. Liu, W. et al. Interference of silibinin with IGF-1R signalling pathways protects human epidermoid carcinoma A431 cells from UVB-induced apoptosis. Biochem Biophys Res Commun 432, 314-9 (2013).

57. Cao, X. et al. In vitro release and in vitro-in vivo correlation for silybin meglumine incorporated into hollow-type mesoporous silica nanoparticles. Int $J$ Nanomedicine 7, 753-62 (2012)

58. Morgillo, F., Woo, J. K., Kim, E. S., Hong, W. K. \& Lee, H. Y. Heterodimerization of insulin-like growth factor receptor/epidermal growth factor receptor and induction of survivin expression counteract the antitumor action of erlotinib. Cancer Res 66, 10100-11 (2006).

59. Shien, K. et al. Acquired resistance to EGFR inhibitors is associated with a manifestation of stem cell-like properties in cancer cells. Cancer Res. 73, 3051-61 (2013).

\section{Acknowledgements}

This work was supported by a charity collection organized by the Fundació Roses Contra el Cáncer (Roses, Girona, Catalonia). This work was also financially supported by the Instituto de Salud Carlos III (Ministerio de Sanidad y Consumo, Fondo de Investigación Sanitaria (FIS), Spain, grants CP05-00090, PI06-0778 and RD06-0020-0028), the Fundación Científica de la Asociación Española Contra el Cáncer (AECC, Spain), and the Ministerio de Ciencia e Innovación (SAF2009-11579 and SAF2012-38914, Plan Nacional de I + D + i, MICINN, Spain). Alejandro Vazquez-Martin received a Sara Borrell post-doctoral contract (CD08/00283, Ministerio de Sanidad y Consumo, Fondo de Investigación Sanitaria -FIS-, Spain). Sílvia Cufí received a research fellowship (BES-2010-032066, Formación de Personal Investigador, FPI) from the Ministerio de Ciencia e Innovación (MICINN, Spain).

\section{Author contributions}

J.A.M., J.B.-B., V.M. and A.V.M. designed the study. S.C., C.O.F., A.V.M., V.Z.T.-G., B.C.F., R.B., E.B.-C. and E.C. performed the experiments. J.A.M., B.M.C., J.B.-B. and J.V. analysed the data. J.A.M. wrote the paper. 


\section{Additional information}

Supplementary information accompanies this paper at http://www.nature.com/ scientificreports

Competing financial interests: AstraZeneca (Spain) provided partial financial support for the present study via an educational grant to J.A.M. and J.B.-B.

How to cite this article: Vazquez-Martin, A. et al. IGF-1R/epithelial-to-mesenchymal transition (EMT) crosstalk suppresses the erlotinib-sensitizing effect of EGFR exon 19 deletion mutations. Sci. Rep. 3, 2560; DOI:10.1038/srep02560 (2013).

(c) (i) (-) This work is licensed under a Creative Commons AttributionCommercial-NoDerivs 3.0 Unported license. To view a copy of this license, visit http://creativecommons.org/licenses/by-nc-nd/3.0 